\title{
Estudo de um sistema de reação-difusão com condições de fronteira não-lineares
}

\author{
Antonio Sergio Munhoz \\ Dissertação Apresentada \\ ao \\ Instituto de Matemática e Estatística \\ da \\ Universidade de São Paulo \\ para Obtenção do Grau \\ de \\ Mestre em Matemática Aplicada
}

Área de Concentração: Equações Diferenciais Parciais Orientador: Prof. Dr. DANIEL BAUMAN HENRY

Durante a elaboração deste trabalho, o autor recebeu apoio financeiro do CNPq.

-São Paulo, novembro de 1998- 


\title{
Estudo de um sistema de reação-difusão com condições de fronteira não-lineares
}

\author{
Antonio Sergio Munhoz
}

Este exemplar corresponde à redação final da dissertação corrigida, defendida por Antonio Sergio Munhoz e aprovada pela comissão julgadora.

São Paulo, 9 de dezembro de 1998.

Banca examinadora:

- Prof. Dr. Daniel Bauman Henry (orientador) - IME-USP

- Prof. Dr. Antonio Luiz Pereira - IME-USP

- Prof. Dr. Alexandre Nolasco de Carvalho - ICMC-USP 
Dedico este trabalho a quem sofreu medo comigo diante do impossível e do incerto, mas manteve, sempre com muito discernimento. a indispensável conviccão no possível.

À Solange, com amor. 


\section{AGRADECIMENTOS}

Ao professor Dan Henry que sempre esteve disposto a pesquisar e resolver todas as dúvidas e obstáculos que foram surgindo no nosso caminho.

Aos professores Adilson, Hamilton, Iracema, Saulo e Sérgio que em diferentes etapas da minha vida acadêmica contribuiram para a minha formação e permanência no IME/USP.

Ao professor Marcelo Mourão da Poli/metalurgia por ter me introduzido na sua área de trabalho e pelas indicações dadas.

Ao amigo engenheiro metalúrgico Ronaldo Mathiazzi por ter me levado com grande entusiasmo ao problema que deu origem a esta dissertação e ao Alexandre M. da Costa (cat's) por ter me ouvido e incentivado sempre.

Ao Fábio, Young, Beth, Victor (ao mestre), Calixto, Miguel que me deram o prazer de suas companhias.

À minha família, pelo apoio incondicional. 


\section{Resumo}

Estudamos um sistema de reação-difusão com condições de fronteira nãolineares

$$
\begin{aligned}
\frac{\partial u^{j}}{\partial t} & =\operatorname{div}\left(D^{j}\left(u^{1}\right) \operatorname{grad} u^{j}\right)+F^{j}(\mathrm{u}) \mathrm{em}\left(t_{0}, t_{1}\right] \times \Omega, \\
\frac{\partial u^{j}}{\partial N} & =g^{j}\left(u^{1}, u^{j}\right) \mathrm{em}\left(t_{0}, t_{1}\right] \times \partial \Omega, \\
u^{j}\left(t_{0}, x\right) & =u_{0}^{j}(x) \mathrm{em} \Omega, \quad j=1, \ldots m,
\end{aligned}
$$

onde $\Omega \subset \mathbb{R}^{n}$ é um aberto limitado com fronteira regular, $\frac{\partial}{\partial N}$ a derivada normal exterior a $\partial \Omega$ e $D^{j} \geq c_{0}>0$.

Damos condições concretas sobre $F^{j}, g^{j}, D^{j}$ e $u_{0}^{j}$ para garantir existência e unicidade de solução local no tempo. Em especial, garantindo também, em certo sentido, continuidade da solução com relação ao dado inicial.

\section{Abstract}

We study a reaction-difusion system with non-linear boundary conditions

$$
\begin{aligned}
\frac{\partial u^{j}}{\partial t} & =\operatorname{div}\left(D^{j}\left(u^{1}\right) \operatorname{grad} u^{j}\right)+F^{j}(\mathbf{u}),(t, x) \in\left(t_{0}, t_{1}\right] \times \Omega, \\
\frac{\partial u^{j}}{\partial N} & =g^{j}\left(u^{1}, u^{j}\right),(t, x) \in\left(t_{0}, t_{1}\right] \times \partial \Omega, \\
u^{j}\left(t_{0}, x\right) & =u_{0}^{j}(x), x \in \Omega, \quad j=1, \ldots, m,
\end{aligned}
$$

where $\Omega \subset \mathbb{R}^{n}$ is a bounded open set with regular boundary, $\frac{\partial}{\partial N}$ the outer normal derivative on the boundary $\partial \Omega$ and $D^{j} \geq c_{0}>0$.

We give concrete conditions about $F^{j}, g^{j}, D^{j}$ and $u_{0}^{j}$ to get the existence and unicity of the local classical solution. In addition. we obtain also, in some sense, the continuity of the solution with respect to inicial condition. 


\section{Apresentação}

Não é difícil construir complexos modelos matemáticos para os chamados sistemas de reação-difusão muito comuns, por exemplo, na engenharia química. Entretanto, responder a uma pergunta básica como se o modelo matemático (dado por sistema de equações diferenciais parciais e condições de fronteira) tem solução é impensável a um não-especialista, mesmo para modelos matemáticos relativamente simples.

Nosso objetivo é esclarecer um pouco esse ponto, pelo menos para certos tipos de modelos matemáticos de sistemas de reação-difusão.

Um aspecto não pouco relevante é que hosso tratamento inclui certos modelos matemáticos com condição de fronteira não-linear(por exemplo, a lei de Stefan-Boltzman que matematiza a troca de calor entre duas superfícies como proporcional a diferença da quarta potência da temperatura de cada superfície). Damos um exemplo explícito de um modelo assim, que, de resto, foi o que originou todo o presente trabalho.

O leitor vai notar que no capítulo que traz a dedução de tal modelo, as aproximações são feitas com pouca fundamentação quantitativa, porque esta só vem à posteriori com o modelo. Um purista pode omitir este capítulo sem prejuizo à estrutura do resto do texto.

Esperamos que este trabalho seja um estímulo à construção de modelos matemáticos para sistemas de reação-difusão mais precisos e compatíveis com a realidade e que clarifique um pouco essa complexa área da matemática aplicada. 


\section{Sumário}

1 Introdução $\quad 8$

2 Modelo físico $\quad 13$

2.1 Pelota autoredutora . . . . . . . . . . . . . . 15

2.2 Reações químicas . . . . . . . . . . . . . . . . . . . . 17

2.3 Balanço de energia . . . . . . . . . . . . . . . . . . 18

2.3.1 Fluxo de calor no interior . . . . . . . . . . . . 18

2.3.2 Fluxo de calor para a superfície . . . . . . . . . . 20

2.4 Balanço de massa . . . . . . . . . . . . . . . . . 20

2.4.1 Fluxo gasoso no interior . . . . . . . . . . . 20

2.4.2 Fluxo gasoso para o exterior . . . . . . . . . . 21

3 Mudança de variável $\quad 23$

3.1 Mudança de variável . . . . . . . . . . . . . . . . . . . . 24

3.1.1 Condições . . . . . . . . . . . . . . . . . . 24

3.1 .2 Construção . . . . . . . . . . . . . . . . 25

4 Resultados de Sobolevskiĭ e aplicação 31

4.1 Equação parabólica não-linear . . . . . . . . . . . . . . . 32

4.1.1 Teorema de existêcia e unicidade . . . . . . . . . . . 32

4.1 .2 Regularização . . . . . . . . . . . . . . . . 33

4.1.3 Dependência em relação ao dado inicial . . . . . . . . 35

4.2 Aplicação . . . . . . . . . . . . . . . . . . . . . . 38

4.2.1 Retorno ao modelo físico . . . . . . . . . . . . 44

5 Conclusão 46

A Aplicação do princípio do máximo $\quad 47$ 


\section{Capítulo 1}

\section{Introdução}

Estudamos uma certa classe de sistemas de reação-difusão visando propor condições suficientes e simples para garantir existência e unicidade de solução local no tempo a um sistema pertencente a tal classe.

A designação sistema de reação-difusão tem dois sentidos complementares. Refere-se a um fenômeno real governado, em termos bem gerais, por leis de transporte e leis de geração ou consumo e, também, ao modelo matemático associado a tal fenômeno. Vamos nos referir a cada sentido, respectivamente, como sistema físico de reação-difusão (SFRD) e sistema matemático de reação-difusão (SMRD). Muitas considerações concernentes a obtenção de SMRD para certos tipos de (SFRD) podem ser achadas em Fife [3] que também contém exemplos de SMRD em diversas áreas, como genética, disseminação de doenças, etc.; Henry [5], especialmente o cap.2, e Rothe [4] também tem muitos exemplos.

Os SFRD e SMRD são o ingrediente essencial de muitos problemas de engenharia química. Os SMRD resultam naturalmente da aplicação das leis de conservação de massa e energia ao SFRD. As leis de conservação, as leis de fluxo de massa ou calor mais as equações de velocidades de reações químicas e as chamadas equações constitutivas completam o SMRD de um típico SFRD da engenharia química.

No cap 2, fazemos a dedução do SMRD do SFRD da engenharia química (e metalúrgica) que motivou nosso estudo a qual foi baseada em Szekelly [16], Sun [17] e Bird [19]. Este SFRD constitui-se de uma pelota composta de grãos de minério de ferro e grãos de carvão submetida a aquecimento em forno elétrico. Como é sabido, a temperaturas suficientemente altas, a mistura dos componentes da pelota torna-se reativa levando a geração de gases e 
formação do ferro metálico que se deseja produzir. Fomos motivados pelo desejo de construir um SMRD que previsse os dados experimentais do SFRD em questão que foram determinados na tese do professor Mourão([15]). Entre estes acham-se medidas de concentração dos gases, temperatura do centro e da superfície da pelota em função de parâmetros diversos, tais como diâmetro da pelota, temperatura do forno, etc.

O SMRD obtido no cap. 2 é um exemplo típico da classe de sistemas matemáticos de reação-difusão que vamos considerar a qual tem a forma

$$
\begin{aligned}
\frac{\partial u^{j}}{\partial t}(t, x)= & \operatorname{div}\left(D^{j}\left(u^{1}(t, x)\right) \operatorname{grad} u^{j}(t, x)\right)+F^{j}(\mathbf{u}(t, x)) \\
& (C F) \\
& (C 0) \quad j=1, \ldots, m
\end{aligned}
$$

ou seja, é um sistema de $m$ equações de equações diferenciais parciais de segunda ordem nas variáveis independentes $t$ e $x$ e nas variáveis dependentes $u^{j}$ ou na variável dependente

$$
\mathbf{u}=\left(\begin{array}{l}
u^{1} \\
\vdots \\
u^{m}
\end{array}\right)
$$

A variável $t$, o tempo, varia no intervalo $\left(t_{0}, t_{1}\right]$ e a variável $x$, a posição, varia no conjunto aberto limitado $\Omega \in \mathbb{R}^{n}$. Como é usual,

$$
\operatorname{grad} u^{j}=\left(\begin{array}{l}
\frac{\partial u^{j}}{\partial x_{1}} \\
\vdots \\
\frac{\partial u^{j}}{\partial x_{n}}
\end{array}\right)
$$

$\mathrm{e}$

$$
\operatorname{div}\left(D^{j}\left(u^{1}\right) \operatorname{grad} u^{j}\right)=\sum_{i=1}^{n} \frac{\partial}{\partial x_{i}}\left(D^{j}\left(u^{1}\right) \frac{\partial u^{j}}{\partial x_{i}}\right) .
$$

Vamos definir adiante a condicão de fronteira $(C F)$ e a condição inicial $(C 0)$.

No SMRD do cap. $2, D^{j}$ representa o coeficiente de condutividade equivalente de calor ou o coeficiente de difusividade equivalente de massa quando estiver na equação de balanço de calor ou massa, respectivamente. Assumimos 
que estes coeficientes satisfazem a chamada condição de parabolicidade,isto é, para qualquer $j, 1 \leq j \leq m$,

$$
D^{j} \geq c_{0}>0, c_{0} \text { constante, }
$$

em todo seu domínio. Existem estudos de certas classes de sistemas de reação-difusão não assumindo a priori tal condicão; tais sistemas são chamados sistemas parabólicos degenerados ([10]).

Observamos que no sistema (1.1) $D^{j}$ depende unicamente de $u^{1}$, que é a temperatura no modelo do cap. 2 , onde há uma explicação física para tal tipo de dependência.

A forma vetorial de (1.1) é

$$
\frac{\partial \mathbf{u}}{\partial t}=\operatorname{div} \mathcal{D} \operatorname{grad} \mathbf{u}+\mathbf{F}(\mathbf{u})
$$

onde $\mathcal{D}$ é a matriz diagonal $\left(\begin{array}{ccc}D^{1} & & \\ & \ddots & \\ & & D^{m}\end{array}\right), \mathbf{F}=\left(\begin{array}{l}F^{1} \\ \vdots \\ F^{m}\end{array}\right)$ e $\frac{\partial \mathbf{u}}{\partial t}=$ $\left(\begin{array}{l}\frac{\partial u^{1}}{\partial t} \\ \vdots \\ \frac{\partial u^{m}}{\partial t}\end{array}\right)$ e

$$
\operatorname{div} \mathcal{D} \operatorname{grad} \mathbf{u}=\left(\begin{array}{l}
\operatorname{div} D^{1} \operatorname{grad} u^{1} \\
\vdots \\
\operatorname{div} D^{m} \operatorname{grad} u^{m}
\end{array}\right)
$$

Como consequência da dependência de $\mathcal{D}$ com a solução $u^{1}$ o sistema (1.2) é dito não-linear. Supondo as funções deriváveis,

$$
\operatorname{div} D^{j} \operatorname{grad} u^{j}=\sum_{i=1}^{n} D_{u^{1}}^{j} \frac{\partial u^{1}}{\partial x_{i}} \frac{\partial u^{j}}{\partial x_{i}}+\sum_{i=1}^{n} D^{j} \frac{\partial^{2} u^{j}}{\partial x_{i}^{2}}
$$

segue que o sitema (1.2) é linear com respeito às derivadas de ordem mais alta (igual a 2) de $u^{j}$. Este tipo de sistema não-linear recebe o nome de quaselinear.

Para completar a formulação matemática do sistema, é preciso dizer também a condição inicial e a condição de fronteira. 
A condição inicial define o valor da variável $\mathbf{u}$ no instante $t=t_{0}$ em todo domínio $\Omega$,

$$
\mathrm{u}\left(t_{0}, x\right)=\mathrm{u}_{0}(x) \text { para } x \in \Omega
$$

onde $u_{0}$ é dado.

A condição de fronteira define o valor de $u$ ou da derivada de $u$ na fronteira de $\Omega$ durante o intervalo $\left(t_{0}, t_{1}\right]$.

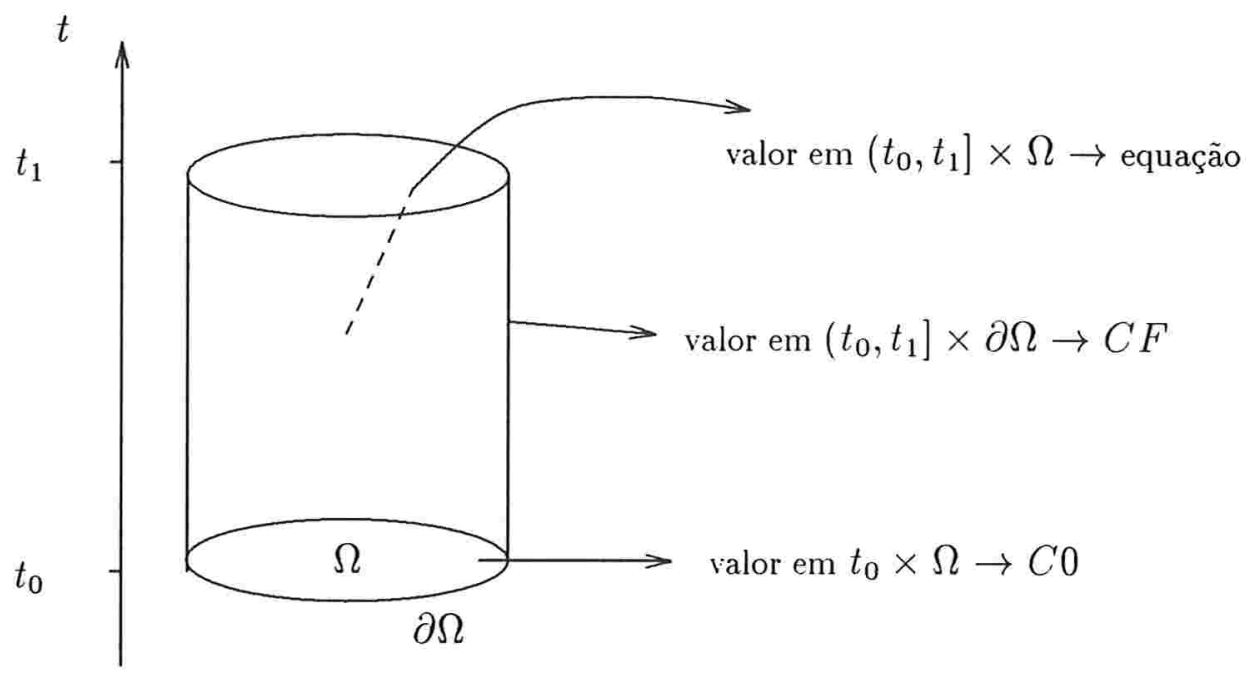

Figura 1.1: Formulação matemática do sistema de reação-difusão em análise.

O sistema (1.2), (1.3) com condição de fronteira de Dirichlet

$$
\mathbf{u}(x)=0, x \in \partial \Omega,
$$

e coeficientes $D^{1}=\ldots=D^{m}$ satisfazendo algumas restrições de crescimento são estudados em Ladyzenskaya [1] (teorema 7.1, p.598), onde há um resultado de existência e unicidade de solução clássica não somente local no tempo, mas também em todo intervalo $\left[t_{0}, t_{1}\right]$.

Sistemas de reação-difusão com matriz $\mathcal{D}$ e condição de fronteira só dependendo de $t$ e $x$ e $\mathbf{F}$ dependendo de $t, x, \mathbf{u}$ e derivadas de ordem 1 de $\mathbf{u}$ são chamados semilineares. Estes sistemas são estudados por Fife [3], Rothe [4] e Henry [5].

Vamos estudar uma classe de sistemas de reação-difusão-(1.2) e (1.3)-com condição de fronteira não-linear do tipo de Neumann

$$
\frac{\partial u^{j}}{\partial N}(t, x)=g^{j}\left(u^{1}(t, x), u^{j}(t, x)\right) \text { em }\left(t_{0}, t_{1}\right] \times \Omega .
$$


onde $\frac{\partial}{\partial N}$ é a derivada na direção normal exterior a $\partial \Omega$. Note a existência de um acoplamento entre $u_{1}$ e $u_{j}$ na condição de fronteira para a variável $u^{j}$. Conforme vai ficar claro, a técnica matemática usada para tratar o problema (através de mudança de variável) depende do fato de o acoplamento na fronteira ter tal restrição. Apesar de ser possível enfraquecê-la (como utilizar acoplamento triangular na fronteira, isto é, $g^{1}\left(u^{1}\right), g^{2}\left(u^{1}, u^{2}\right), g^{3}\left(u^{1}, u^{2}, u^{3}\right)$, etc.)e ainda assim utilizar a mesma técnica, por simplicidade, vamos considerar somente o acoplamento acima que de resto é suficiente à aplicação ao modelo matemático do sistema de reação-difusão que vamos enfocar. Isto não significa que menosprezemos a importância em estender a aplicação da técnica mencionada, pelo contrário, cremos que sua extensão pode trazer ainda maior clareza ao assunto.

O uso de condição de fronteira não-linear com o acoplamento acima decorre de uma necessidade física (cap. 2). No entanto, seu uso em modelagem matemática de problemas de engenharia química não parece muito comum, provavelmente devido à grande complexidade matemática associada. Foi o prof. Henry quem propôs que estudássemos o sistema (1.2), (1.3) com tal tipo de condição de fronteira.

Parece que o grande estudioso dos sistemas de equaçòes diferenciais parciais parabólicas quaselineares de segunda ordem com condição de fronteira não-linear - dos quais o sistema (1.2), (1.3) e (1.4) é um caso particular - é Herbert Amann [6].

O método usado aqui tem certa semelhança com o de Amann. Em ambos, o sistema é visto como uma equação ordinária em algum espaço de Banach convenientemente escolhido para aplicação dos resultados de Soboleviskiř [8]. Entretanto, existe uma diferença fundamental entre os métodos. Amann aplica diretamente os resultados de Soboleviskï no contexto de um espaço de Banach cujos elementos satisfazem (1.4) em algum sentido que não entendemos bem qual é (através do uso dos chamados espaços de extrapolação), enquanto aqui primeiramente vamos fazer uma mudança de variável (cap.3) que transforma o sistema (1.2), (1.3) e (1.4) em um sistema equivalente com condição de fronteira muito mais simples-(3.21)-e só aí aplicamos os resultados de Soboleviskiu (cap. 4).

Através dos resultados de Sobolevskiı̌ e resultados de regularidade, vamos chegar ao objetivo principal desta dissertação, ou seja, provar que os sistemas (1.2)-(1.4)-(1.3), sob certas simples condições, tem realmente solução $\mathbf{u}$, sendo suas componentes $u^{j}(t, x)$ superfícies lisas no domínio do tempo-espaço. 


\section{Capítulo 2}

\section{Modelo físico}

Vamos considerar neste capítulo o problema de modelar matematicamente um exemplo concreto de sistema de reação-difusão. Nos termos da introdução, vamos obter o SMRD de um certo SFRD.

Esse sistema é uma pelota esférica porosa, constituída por pequenos grãos de hematita $\left(\mathrm{Fe}_{2} \mathrm{O}_{3}\right)$ e grãos de carvão $(\mathrm{C})$ exposta a aquecimento (queimada) em um forno elétrico(figura 2.1) sob uma atmosfera circundante de gás inerte(p.ex. nitrogênio). Muitos reações químicas ((2.1), (2.2), (2.3), (2.4)) sólido-gás tem lugar na pelota se a temperatura do forno é suficentemente grande.
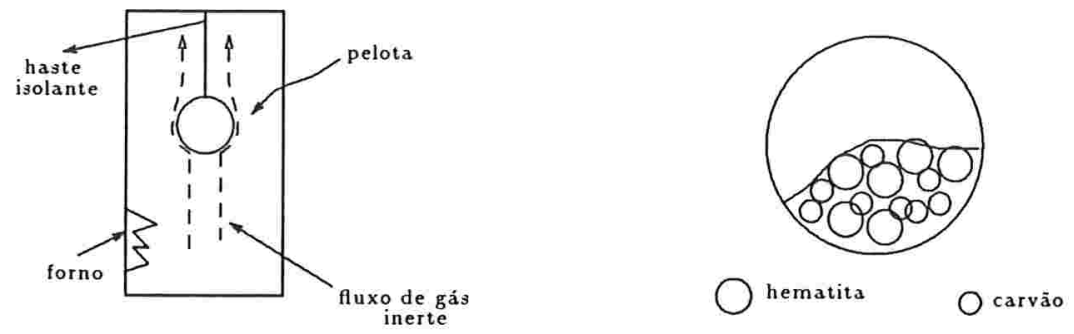

Figura 2.1: Esquema do sistema modelado e esquema da pelota autoredutora

Uma das nossas motivações é construir um modelo matemático sem assumir que a temperatura em qualquer ponto da pelota seja permanentemente igual à do forno, isto é, supor sistema isotérmico. Tal hipótese, embora comumente feita por muitos estudiosos(p.ex.Szekelly [16]) em sistemas similares, não é confirmada pelos dados experimentais obtidos por Mourão (gráfico 2.2).

Entre as reações químicas que ocorrem na pelota, aquela conhecida co- 


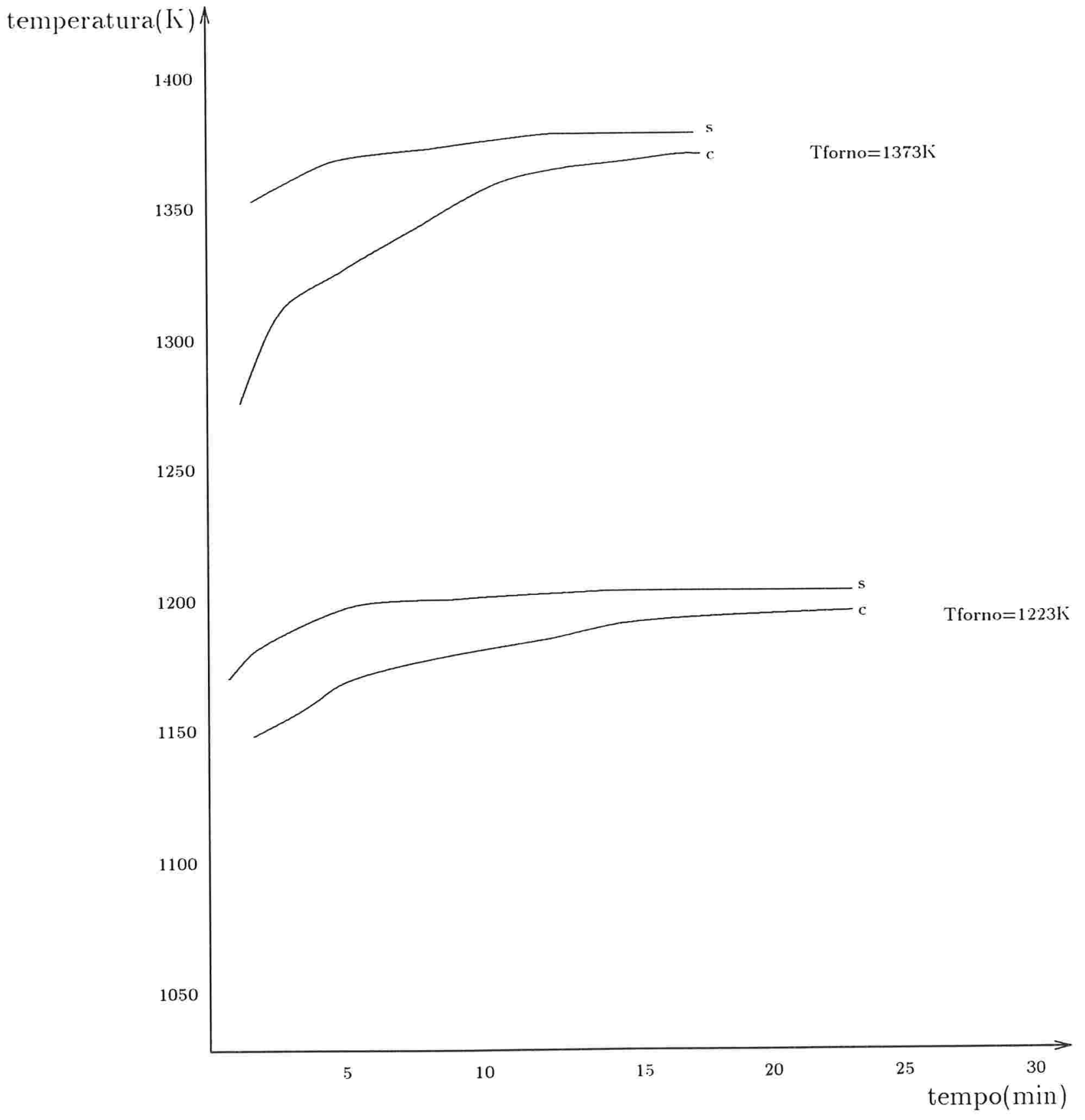

Figura 2.2: Temperatura na superfície(s) e no centro(c) da pelota. 
mo reação de Boudward ou gaseificação do carbono(equação (2.1)) é muito endotérmica e portanto a sua ocorrência nas camadas mais externas da pelota consome uma parte do calor que chega à pelota, o que diminui o fluxo de calor para as camadas mais internas. Tem-se aí uma possível explicação qualitativa para existência de um não-desprezível gradiente de temperatura na pelota (gráfico 2.2).

Neste capítulo, vamos propor um modelo matemático como um incipiente tratamento quantitativo com vista ao cálculo do perfil de temperatura e concentração dos gases no interior e na superfície da pelota.

\subsection{Pelota autoredutora}

Como dito antes, a pelota que vai ser queimada no forno (figura 2.1) é constituida por grãos de hematita (minério de ferro) e por grãos de carvão. Vamos também supor que os grãos dos dois sólidos tenham forma esférica e os grãos de cada sólido tenham diâmetros iguais.

Para preparação destas pelotas, o minério de ferro e o carvão são moídos separadamete até atingirem uma granulometria desejada e depois introduzidos conjuntamente em um misturador até obter uma mistura homogênea. Com esta massa uniforme, fazemos manualmente pelotas com formato esférico,composição e diâmetro de grãos definidos, como por exemplo a da tabela (2.1).

Tabela 2.1: Exemplo de pelota

$\begin{array}{ll}\text { Diâmetro da pelota } & 1,6 \mathrm{~cm} \\ \text { Proporção molar } & 1 \mathrm{Fe}_{2} \mathrm{O}_{3}: 3 \mathrm{C} \\ \text { Diâmetro dos grãos de } \mathrm{Fe}_{2} \mathrm{O}_{3} & 5,95.10^{-4} \mathrm{~cm} \\ \text { Diâmetro dos grãos de C } & 4,25 \cdot 10^{-4} \mathrm{~cm} \\ \text { Densidade medida da pelota } & 2,066 \mathrm{~g} / \mathrm{cm}^{3} \\ \text { Densidade verdadeira de } \mathrm{Fe}_{2} \mathrm{O}_{3} & 5,24 \mathrm{~g} / \mathrm{cm}^{3} \\ \text { Densidade verdadeira de C } & 1,52 \mathrm{~g} / \mathrm{cm}^{3}\end{array}$

A tabela 2 traz também as densidades verdadeiras do minério e do carvão usados para preparar a pelota. Esta é a densidade destes sólidos sob forma compacta.

Vamos calcular a porosidade e o número de grãos de cada tipo por $\mathrm{cm}^{3}$ da pelota dada na tabela 2.1. 
Conhecida a proporção molar. como $\mathrm{mol} \mathrm{Fe}=56$, mol $\mathrm{C}=12$, mol $\mathrm{O}=16$, temos as frações de massa de $\mathrm{C}\left(=x_{1}\right)$ e de $\mathrm{Fe}_{2} \mathrm{O}_{3}\left(=x_{2}\right)$,

$$
x_{1}=\frac{3(12)}{3(12)+2 .(56)+3 .(16)}=18,4 \% \text { e } x_{2}=81,6 \% .
$$

Então $1 \mathrm{~g}$ de pelota tem $0,184 \mathrm{~g}$ de $\mathrm{C}$ e $0,816 \mathrm{~g}$ de $\mathrm{Fe}_{2} \mathrm{O}_{3}$ que ocupam espaço $(0,184 / 1,52+0,816 / 5,24) \mathrm{cm}^{3}=(1 / 3,61) \mathrm{g} / \mathrm{cm}^{3}$ ou seja, a densidade 'compactada' seria $3,61 \mathrm{~g} / \mathrm{cm}^{3}$, enquanto a densidade medida é de fato $2,066 \mathrm{~g} / \mathrm{cm}^{3}$. A fração do volume ocupado é $2,066 / 3,61=0,57$ e a porosidade $\epsilon=1-2,066 / 3,61=0,43$.

Chamando $f_{1}$ e $f_{2}$ a fração volumétrica ocupada, respectivamente, por $\mathrm{C}$ e $\mathrm{Fe}_{2} \mathrm{O}_{3}$ em relação ao volume tolal ocupado, temos

$$
\frac{f_{1}}{f_{2}}=\frac{0,184 / 1,52}{0,816 / 5,24}=0,777 .
$$

Usando também $f_{1}+f_{2}=1$, obtemos $f_{1}$ e $f_{2}$.

Como a fração de volume total ocupado em relação ao volume da pelota vale $1-\epsilon$, a fração volumétrica de 1 e 2 em relação ao volume total da pelota são, respectivamente, $f_{1}(1-\epsilon)$ e $f_{2}(1-\epsilon)$. Resulta a distribuição volumétrica na pelota (tabela 2.2).

Tabela 2.2: Distribuição volumétrica na pelota

$$
\begin{array}{ll}
\text { Porosidade } & 0,43 \\
\text { Fração volumétrica de C } & 0,25 \\
\text { Fraçao volumétrica de } \mathrm{Fe}_{2} \mathrm{O}_{3} & 0,32
\end{array}
$$

Finalmente, o cálculo do número de grãos de 1 por $\mathrm{cm}^{3}\left(N_{1}\right)$ e de $2\left(N_{2}\right)$

$$
\begin{aligned}
& N_{1}=\frac{0,25}{\pi / 6 \cdot\left(4,25 \cdot 10^{-4}\right)^{3}}=6,22 \cdot 10^{9} \text { grãos de } 1 / \mathrm{cm}^{3} \\
& N_{2}=\frac{0,32}{\pi / 6\left(5,95 \cdot 10^{-4}\right)^{3}}=2,9 \cdot 10^{9} \text { grãos de } 2 / \mathrm{cm}^{3} .
\end{aligned}
$$

Com estes dados é possivel calcular, por exemplo, a área superfícial dos sólidos 1 e 2 . 


\subsection{Reações químicas}

Se a temperatura do forno for suficientemente alta a mistura porosa da pelota torna-se reativa, tendo início muitas reaçòes químicas (veja diagrama termodinâmico 2.3, extraído de [16], fig.5.2, p.181)

$$
\begin{gathered}
\mathrm{C}_{(s)}+\mathrm{CO}_{2(g)}=2 \mathrm{CO}_{(g)} \\
3 \mathrm{Fe}_{2} \mathrm{O}_{3(s)}+\mathrm{CO}_{(g)}=2 \mathrm{Fe}_{3} \mathrm{O}_{4(s)}+\mathrm{CO}_{2(g)} \\
\mathrm{Fe}_{3} \mathrm{O}_{4(s)}+\mathrm{CO}_{(g)}=3 \mathrm{FeO}(s)+\mathrm{CO}_{2(g)} \\
F \epsilon O_{(s)}+\mathrm{CO}_{(g)}=F \epsilon_{(s)}+\mathrm{CO}_{2(g)}
\end{gathered}
$$

simultâneas e acopladas entre si. Sào reaçòes de reduçào que procedem através de intermediários gasosos. heterogêneas sólido-gás com expressōes cinéticas dependendo da temperatura, pressào parcial dos gases, área interfacial dos sólidos.

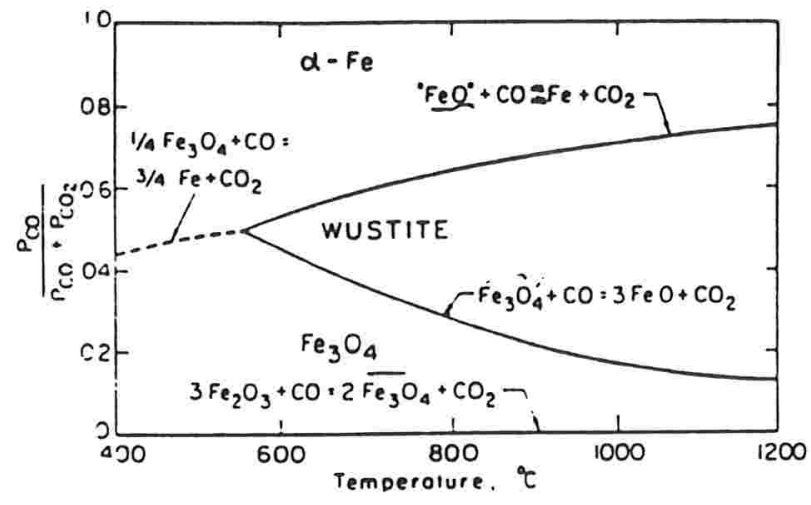

Figura 2.3: Diagrama de equilíbrio para óxidos de ferro na presença de misturas de monoxido de carbono e dióxido de carbono 
Existem muitas hipóteses relativas à inicialização deste processo. Vamos supor a concentração inicial de $\mathrm{CO}$ e $\mathrm{CO}_{2}$ na pelota igual a destes gases no meio ambiente.

Desprezemos a influência da variação da área interfacial dos sólidos com o curso da reação nas expressões de velocidade. Isto implica que vamos considerar somente a cinética das reações de Boudward (2.1) e de redução da hematita (2.2).

Podemos assumir em geral que velocidades das reações químicas sejam lineares com as concentrações dos gases, com constantes de reação dependendo da temperatura segundo a lei de Arrhenius

$$
k=k_{0} e^{\frac{-E}{R T}}
$$

onde $E$ é a energia de ativação da reação, $R$ a constante universal e $T$ a temperatura no sítio de reação.

Em particular, a cinética do sentido da esquerda para direita da reação de Boudward (2.1) é mais convenientemente expressa por uma lei do tipo Langmuir-Hinshelwood ([16], p.152)

$$
\mathcal{R}=\frac{k c_{1}}{1+K c_{1}}
$$

onde $\mathcal{R}$ é a velocidade local em moles de 1 por unidade de superfície por unidade de tempo, $c_{1}$ a concentração de reagente 1 na superfície de reação(no caso, $1=\mathrm{CO}_{2}$ ) e $k, \mathrm{~K}$ as constantes cinéticas de Langmuir-Hinshelwood.

Por razões termodinâmicas, abaixo de uma certa temperatura $T_{R}$, a mistura que constitui a pelota torna-se não-reativa e, portanto,

$$
\mathcal{R}=0 \text { se } T \leq T_{R} .
$$

Para o ponto de vista do presente trabalho, não é necessário conhecer explicitamente as leis cinéticas das reações químicas. É suficiente admitir a dependência destas leis com a concentração de gases e temperatura suficientemente regular.

\subsection{Balanço de energia}

\subsubsection{Fluxo de calor no interior}

O fluxo de calor da superfície para o interior da pelota é, em princípio. devido a vários mecanismos. 
Como o fluxo de gases é das regiões internas mais frias para as regiões externas mais quentes, desprezamos o fluxo de calor por convecção da mistura gasosa. Somos forçados a fazer esta hipótese também porque nada é sabido sobre a pressão total no interior da pelota - a força motriz do fluxo convectivo. Daí, por simplicidade, a pressão total é assumida uniforme no interior da mistura gasosa que ocupa os poros da pelota. Isto também nos livra de considerarmos as equações de movimento de Navier-Stokes para estudar o perfil de pressão e mantém o modelo mais ou menos tratável.

A condutividade dos gases é muito menor do que a condutividade dos sólidos da pelota. Portanto, desprezamos o fluxo de calor por condução através da mistura gasosa.

O fluxo de calor $\left(N_{1}\right)$ é, pela lei de Fourier,

$$
N_{1}=-k_{s}(T)(1-\epsilon) \operatorname{grad} T,
$$

[somente condução no sólido; gás e sólido localmente em equilíbrio] onde $\epsilon$ é a porosidade da pelota, $k_{s}$ é a condutividade dos sólidos obtida ponderando pela fração volumétrica a condutividade de cada sólido que constitui a pelota. Vamos considerar somente a dependência da condutividade de cada sólido com a temperatura $T$ (em [18], a condutividade da hematita densa é dada por uma expressão do tipo $\frac{1}{a T+b}$, a,b constantes positivas), supondo a porosidade e fraçoes volumétricas de sólidos constantes. A expressão usada para condutividade efetiva no interior do material poroso: $(1-\epsilon) k_{s}$ é válida quando os poros são isolados entre si ([20], relação (6.25), p. 199); assumimos ser esse o nosso caso. Para poros grandes e não isolados, a porosidade torna-se contínua e o cálculo da condutividade é um pouco mais complexo ([20]. p.200).

As velocidades das reações químicas entre os gases que ocupam os poros da pelota com a superfície dos sólidos depende da temperatura (relação 2.5). Por simplicidade, a temperatura dos gases que envolvem cada grão é assumida igual a da superfície do mesmo grão. tem-se

Evidenciando cada termo do balanço de calor (ou melhor, de entalpia),

$$
\left(\rho_{s}(1-\epsilon) C_{p_{s}}+\epsilon \rho_{g} C_{p_{g}}\right) \frac{\partial T}{\partial t}=\operatorname{div} k_{s}(T)(1-\epsilon) \operatorname{grad} T-\left(\sum \mathcal{R}_{i} \Delta H_{i}\right) .
$$

$\rho_{s}, C_{p_{s}}$ denotam, respectivamente a densidade e capacidade térmica dos sólidos; analogamente $\rho_{g}, C_{p_{g}}$ para os gases. Como a densidade dos gases 
é muito pequena em relação a dos sólidos, a segunda parcela do primeiro membro da equação acima é desprezada. Também $C_{p_{s}}$ e $\rho_{s}$ são supostos constantes. Finalmente, $\mathcal{R}_{i}$ é a velocidade da reação química $i$ (seção 2.2) expressa em moles de $i$ por unidade de volume total por unidade de tempo e $\Delta H_{i}$ a variação de entalpia correspondente à reação $i$.

\subsubsection{Fluxo de calor para a superfície}

A recepção de calor do meio envolvente pela superfície da pelota é por radiação, condução pela atmosfera circundante e também por convecção forçada se o gás inerte flui com velocidade diferente de zero. Vamos desprezar o fluxo de calor por condução.

O fluxo na superfície da pelota, com as contribuições devidas à radiação e a conveç̧ão é

$$
-k(T)(1-\epsilon) \frac{\partial T}{\partial N}=\sigma e\left(T^{4}-T_{f}^{4}\right)+h\left(T-T_{f}\right),
$$

onde $\sigma$ é a constante de Stefan-Boltzman, $e$, a emissividade da superfície, $h$, coeficiente de conveç̧ão de calor de Newton, $T_{f}$, a temperatura do forno e $T$, a temperatura em cada ponto da superfície.

O coeficiente de convecção de calor $h$ devido ao fluxo do meio envolvente que tem velocidade $v_{\infty}$ em pontos distantes da pelota pode ser calculado pela relação de W.E.Ranz e W.R. Marshall ([20], relação (8.10), p.250)

$$
h D / k_{f}=2.0+0.60\left(D v_{\infty} \rho_{f} / \mu_{f}\right)^{1 / 2}\left(C_{p} \mu / k\right)_{f}^{1 / 3}
$$

onde $k_{f}, \rho_{f}, \mu_{f}, C_{p}$ designam, respectivamente, a condutividade, densidade, viscosidade e capacidade térmica a pressão constante do gás circunjacente à pelota calculados na temperatura de filme $\left(T_{f}+T\right) / 2$, onde $T_{f}$ é a temperatura do forno e $T$, a temperatura da superfície. Note a presença dos números admensionais $\mathrm{Nu}=h D / k$ (número de Nusselt), $\mathrm{Re}=D v_{\infty} \rho / \mu$ (número de Reynolds) e $\operatorname{Pr}=C_{p} \mu / k$ (número de Prandtl).

\subsection{Balanço de massa}

\subsubsection{Fluxo gasoso no interior}

Os gases fluem pelo interior da pelota por difusào e conveç̧ão. Como o perfil de pressão é assumido uniforme, não há convecçao gasosa e o transporte 
é unicamente por difusão porosa.

Podemos obter um balanço de massa análogo ao balanço de entalpia para cada um dos gases $C O=1, \mathrm{CO}_{2}=2$ no interior da pelota

$$
\frac{\partial \epsilon c_{i}}{\partial t}=\operatorname{div} \frac{\epsilon}{\tau} D^{i}(T) \operatorname{grad} c_{i}+\sum R_{i} \Delta_{i l}
$$

onde $c_{i}$ é a concentração molar da espécie $i=1,2$ por volume vazio, $D^{i}(T)$ a difusividade do gás $i$ na temperatura $T, \tau$, a constante de tortuosidade da mistura granulométrica que constitui a pelota e $\Delta x_{i l}$ o coeficiente estequiométrico do gás $i$ na reação $l$.

Para misturas binárias, como é o caso, admitindo que não haja penetração de gas inerte da atmosfera circunjacente no interior da pelota, as difusividades $D^{1}=D^{2}=D^{1,2}$ podem ser calculados pela fórmula de Chapman-Enskog (Bird[19], p.16-19, relação (16.4-12))

$$
D^{1,2}=0.001858 \frac{\sqrt{T^{3}\left(\frac{1}{M_{1}}+\frac{1}{M_{2}}\right)}}{p \sigma_{1,2}^{2} \Omega_{D ; 1,2}}
$$

onde $M_{1}, M_{2}$ são as massas molares de $1 \mathrm{e} 2$, p, a pressão ambiente e $\sigma_{1,2}$, $\Omega_{D, 1,2}$ são parâmetros definidos em [19], p.16-21, sendo que o primeiro depende somente dos gases 1 e 2 e o segundo depende também da temperatura. Assumimos que tal dependência é regular (veja tabela B-2 de Bird [19]).

A expressão usada para a difusividade efetiva $\frac{\epsilon}{\tau} D^{1}$ na equação $(2.10)$ pode ser achada em Szekelly [16], relação (2.3.20), p.27). Estamos admitindo que os poros da pelota sejam pequenos; para poros grandes e casos intermediários, ver Szekelly [16], p.25.

\subsubsection{Fluxo gasoso para o exterior}

O fluxo de gás para o exterior também é uma lei de fluxo de Newton

$$
-D^{i} \frac{\epsilon}{\tau} \frac{\partial c_{i}}{\partial N}=h\left(c_{i}-c_{i}^{0}\right),
$$

onde $c_{i}^{0}$ é a concentração do gás $i=1,2$ ( na atmosfera circundante (aproximadamente igual a zero), $c_{i}$, a concentração em cada ponto da superfície e $h$, o coeficiente de convecção de massa. 
O coeficiente de convecção de massa devido ao fluxo do gás inerte circunjacente com velocidade distante da pelota igual a $v_{\infty}$ pode se calculado pela expressào de Ranz e Marshall

$$
h D / D^{i}=2.0+0.6\left(D v_{\infty} \rho_{f} / \mu_{f}\right)^{1 / 2}\left(\mu_{f} /\left(\rho_{f} D^{i}\right)\right)^{1 / 3},
$$

onde os parâmetros tem os mesmo sentido de (2.9) e observe a presença do número de Sherwood ( $\left.\mathrm{Sh}=h D / D^{i}\right)$, de Reynolds $\left(\operatorname{Re}=D v_{\infty} \rho / \mu\right)$ e de Schmidt $\left(\mathrm{Sc}=\mu /\left(\rho D^{i}\right)\right)$. 


\section{Capítulo 3}

\section{Mudança de variável}

Como dissemos no cap. 1, pretendemos provar existência de solução para o problema

$$
\begin{aligned}
\frac{\partial u^{j}}{\partial t} & =\operatorname{div}\left(D^{j}\left(u^{1}\right) \operatorname{grad} u^{j}\right)+F^{j}(\mathbf{u}) \mathrm{em}\left(t_{0}, t_{1}\right] \times \Omega, \\
\frac{\partial u^{j}}{\partial N} & =g^{j}\left(u^{1}, u^{j}\right) \operatorname{em}\left(t_{0}, t_{1}\right] \times \partial \Omega, \\
u^{j}(0, x) & =u_{0}^{j}(x) \mathrm{em} \Omega, \quad j=1, \ldots, m,
\end{aligned}
$$

quando as funções $D^{j}, F^{j}, g^{j}, u_{0}^{j}$ satisfazem certas condições.

Estamos chamando solução $\mathrm{u}=\operatorname{col}\left(u_{1}, \ldots, u_{m}\right)$ do problema (3.1)-(3.2)(3.3) a conhecida solução clássica. Neste caso, as derivadas presentes no problema têm o sentido comum.

Definição 3.0.1 Chamamos $\mathbf{u}=\operatorname{col}\left(u_{1}, \ldots, u_{m}\right)$ solução clássica do problema (3.1)-(3.2)-(3.3) se para todo $j, j=1, \ldots, m$, tem-se $u^{j}(t, x)$ contínua em $\left[t_{0}, t_{1}\right] \times \bar{\Omega}$, $\frac{\partial u^{j}}{\partial x_{i}}$ qualquer $i, 1 \leq i \leq n$, contínua em $\left(t_{0}, t_{1}\right] \times \bar{\Omega}, \frac{\partial u^{j}}{\partial t}$ $e \frac{\partial^{2} u^{j}}{\partial x_{i} x_{k}}$, qualquer $i, k, 1 \leq i, k \leq n$, contínuas em $\left(t_{0}, t_{1}\right] \times \Omega$ e u satisfaz (3.1)-(3.2)-(3.3).

Na secção 3.1 .1 dizemos quais são as condições sobre $D^{j}, F^{j}$ e $g^{j}$ que fazem este problema ter solução clássica. Dizemos a condição precisa (e complexa) sobre $u_{0}^{j}$ no capítulo 4 . Assumamos por enquanto que $u_{0}^{j}(x)$ é de classe $C^{2}(\bar{\Omega})$ e satisfaz $(3.2)$ em $t=t_{0}$. 
É bom não nos perdermos pelo longo caminho que temos para percorrer. Não vamos dar neste capítulo a prova de existência, mas por conveniência já damos as condições para isto. Fazemo-la somente no capítulo 4.2.

Relatamos as condições desde já porque precisamos delas para fazermos a prometida mudança de variável mencionada no capítulo 1 . Com esta mudança de variável, vamos transformar o problema (3.1)-(3.2)-(3.3) em um problema equivalente com condição de fronteira linear; este novo problema é descrito por (3.47)-(3.21)-(3.48).

Fazer esta mudança de variável e definir o que estamos entendendo por equivalência constituem os objetivos deste capítulo.

Usamos como referência básica o apêndice de [9] com certas sugestões do próprio prof. Henry.

\subsection{Mudança de variável}

\subsubsection{Condições}

Admitamos que os termos $D^{j}, F^{j}, g^{j}$ e $\mathbf{u}_{0}$ do problema (3.1)-(3.2)-(3.3) satisfaçam as condições abaixo. Para isto, vamos olhar os três primeiros como funções a valores reais tais que o domínio de $D^{j}$ e $F^{j}$ é, respectivamente, $\mathbb{R}$ e $\mathbb{R}^{n}$ e o domínio de $g^{j}$ é $\mathbb{R}$ ou $\mathbb{R}^{2}$ se $j=1$ ou $j \neq 1$. As condições valem para $j=1, \ldots, m$. Finalmente, $\epsilon$ é algum número real tal que $0<\epsilon \leq 1$.

1. (Condição de parabolicidade) $D^{j}(\theta) \geq c_{0}$ para todo $\theta \in \mathbb{R}$ e alguma constante $c_{0}>0$.

2. $\mathbb{R} \ni \theta \rightarrow D^{j}(\theta), C^{1, \epsilon}$.

3. $\mathbb{R}^{m} \ni \theta \rightarrow F^{j}(\theta), C^{0, \epsilon}$.

4. $\mathbb{R} \ni \theta \rightarrow g^{1}(\theta) . C^{2, \epsilon} ; \mathbb{R}^{2} \ni(\phi, \theta) \rightarrow g^{j}(\phi, \theta), C^{2, \epsilon}$ se $j \neq 1$.

5. $\mathrm{u}_{0}(x)=\operatorname{col}\left(u_{0}^{1}(x), \ldots, u_{0}^{m}(x)\right)$ é tal que $u_{0}^{j} \in C^{2}(\bar{\Omega})$ e satisfaz (3.2) em $t=t_{0}$.

Observação 3.1.1 Para tudo o que segue, é suficiente assumir que a regularidade requerida acima para as funções $D^{j}, F^{j}, g^{j}$ verifica-se em algum domínio limitado suficientemente grande. 
Observação 3.1.2 Para definição e propriedades dos espaços de Hölder $C^{m, \epsilon}$, há muitas referências, єm especial, Adams [13], p.9.

Observação 3.1.3 No cap. 4 impomos uma condição mais fraca (e mais complexa) sobre $\mathrm{u}_{0}$.

\subsubsection{Construção}

Para eliminar a não-linearidade da fronteira do problema (3.1)-(3.2)-(3.3), um caminho pode ser construir funções $\varphi^{j}$ que tenham derivada na direção normal à fronteira $\frac{\partial}{\partial N}$ igual a $g^{j}$. Realmente, se as obtivermos podemos definir novas variáveis dependentes chamadas, digamos, de $v^{j}$, em função das velhas $u^{j}$

$$
v^{j}=u^{j}-\varphi^{j} .
$$

Daí tomando a derivada na fronteira $\frac{\partial}{\partial N}$ de ambos os membros de (3.4) temos

$$
\frac{\partial v^{j}}{\partial N}=0
$$

como desejamos.

Para definir $\varphi^{j}$ precisamos do lema 3.1.4.

Lema 3.1.4 Seja $\Omega$ um conjunto aberto limitado de $\mathbb{R}^{n}$ com fronteira $\partial \Omega$ de classe $C^{2, \epsilon}$, com $0<\epsilon \leq 1$. Então existe uma função $C^{2, \epsilon}(\bar{\Omega})$ que chamamos $\xi$ tal que

$$
\begin{aligned}
\xi(x) & =0, x \in \partial \Omega, \\
\frac{\partial \xi}{\partial N}(x) & =-1, x \in \partial \Omega, \\
\xi(x) & >0, x \in \Omega .
\end{aligned}
$$

Note que a mudança de variável para $u^{j}+\xi g^{j}$ já elimina a não-linearidade da fronteira, pois, em $x \in \partial \Omega$,

$$
\frac{\partial}{\partial N}\left(u^{j}+\xi g^{j}\right)=g^{j}+\frac{\partial \xi}{\partial N} g^{j}+\frac{\partial g^{j}}{\partial N} \xi=0 .
$$

Como vai ficar claro adiante, esta função $\xi$ ainda não é completamente conveniente para fazermos a mudança de variável. Dado $\delta>0$ qualquer, vamos definir uma funçào $c_{\delta}$ a partir de $\xi$

$$
c_{\delta}(x)=\delta\left(e^{-\frac{1}{\delta} \xi(x)}-1\right) .
$$


Tem-se que

$$
\begin{aligned}
\left|c_{\delta}(x)\right| & <\delta, x \in \bar{\Omega}, \\
c_{\delta}(x) & =0, x \in \partial \Omega, \\
\frac{\partial c_{\delta}}{\partial N}(x) & =1 .
\end{aligned}
$$

$\operatorname{Sejam} \Psi^{j}, j=1, \ldots, m$, funções reais com domínio $\bar{\Omega} \times \mathbb{R} \subset \mathbb{R}^{n+1}$ se $j=1$ e $\bar{\Omega} \times \mathbb{R}^{2} \subset \mathbb{R}^{n+2}$ se $j \neq 1$ definidas por

$$
\begin{aligned}
\Psi^{1}(x, \theta) & =\theta+g^{1}(\theta) c_{\delta}(x), \\
\Psi^{j}(x, \phi, \theta) & =\theta+g^{j}(\phi, \theta) c_{\delta}(x) .
\end{aligned}
$$

Escolhamos um número $B>0$ qualquer. Como $g^{j}$ é continuamente derivável devido a condição (4) da seção 3.1.1, resulta que no limitado dado por $|\theta| \leq$ $B+1$ se $\mathrm{j}=1$ e por $|\phi| \leq B+1,|\theta| \leq B+1$ se $j \neq 1$, tem-se

$$
\begin{aligned}
& \left|\delta g^{j}\right| \leq 1, \\
& \left|\delta g_{\theta}^{j}\right| \leq k<1 \text { para algum } k>0,
\end{aligned}
$$

se $\delta>0$ é suficientemente pequeno. Fixemos um $\delta$ assim. Então, usando (3.15), (3.16) e (3.10) obtemos que a funções $\Psi^{j}$ satisfazem

$$
\begin{aligned}
& \left|\Psi^{j}-\theta\right| \leq 1, \\
& \left|\Psi_{\theta}^{j}-1\right| \leq k<1,
\end{aligned}
$$

em $x \in \bar{\Omega},|\theta| \leq B+1$ se $j=1$ e em $x \in \bar{\Omega},|\phi| \leq B+1,|\theta| \leq B+1$ se $j \neq 1$.

Finalmente, fazemos a mudança de variável dependente de $u^{j}$ no sistema (3.1), (3.2), (3.3) para $v^{j}$, esta sendo dada implicitamente por

$$
\begin{aligned}
& u^{1}(t, x)=\Psi^{1}\left(x, v^{1}(t, x)\right), \\
& u^{j}(t, x)=\Psi^{j}\left(x, v^{1}(t, x), v^{j}(t, x)\right), \text { se } j \neq 1 .
\end{aligned}
$$

$\mathrm{em}(t, x) \in\left(t_{0}, t_{1}\right] \times \bar{\Omega}$.

Vamos verificar que esta mudança de variável elimina a não-linearidade da fronteira. De fato, de (3.13), (3.11), (3.12), em $x \in \partial \Omega$,

$$
\frac{\partial \Psi^{1}}{\partial N}\left(x, v^{1}\right)=\frac{\partial v^{1}}{\partial N}+g^{1}\left(v^{1}\right)=\frac{\partial v^{1}}{\partial N}+g^{1}\left(u^{1}\right),
$$


e de (3.19) e (3.2),

$$
\frac{\partial v^{1}}{\partial N}=0
$$

A verificação dos demais casos é análoga. Ou seja, para todo $j, 1 \leq j \leq m$,

$$
\frac{\partial v^{j}}{\partial N}=0
$$

Para fazer a substituição de $u^{1}(t, x)=\Psi^{1}\left(t, v^{1}(t, x)\right)$ em $(3.1), \operatorname{com} j=1$, primeiramente usamos a regra da cadeia para obter

$$
\begin{aligned}
\frac{\partial \Psi^{1}}{\partial t}= & \Psi_{\theta}^{1} \frac{\partial v^{1}}{\partial t} \\
\operatorname{grad} \Psi^{1}\left(x, v^{1}\right)= & \operatorname{grad} \Psi^{1}+\Psi_{\theta}^{1} \operatorname{grad} v^{1} \\
\operatorname{grad} D^{1}\left(\Psi^{1}\left(x, v^{1}\right)\right)= & D_{\theta}^{1} \operatorname{grad} \Psi^{1}+D_{\theta}^{1} \Psi_{\theta}^{1} \operatorname{grad} v^{1} \\
\operatorname{div} \operatorname{grad} \Psi^{1}\left(x, v^{1}\right)= & \Delta \Psi^{1}+\operatorname{grad} \Psi_{\theta}^{1} \cdot \operatorname{grad} v^{1} \\
& +\operatorname{grad} \Psi_{\theta}^{1} \cdot \operatorname{grad} v^{1}+\Psi_{\theta \theta}^{1}\left|\operatorname{grad} v^{1}\right|^{2}+ \\
& +\Psi_{\theta}^{1} \Delta v^{1}
\end{aligned}
$$

$\left.\operatorname{div} D^{1}\left(\Psi^{1}\left(x, v^{1}\right)\right) \operatorname{grad} \Psi^{1}\left(x, v^{1}\right)\right)=\operatorname{grad} D^{1}\left(\Psi^{1}\left(x, v^{1}\right)\right) \cdot \operatorname{grad} \Psi\left(x, v^{1}\right)+$ $D^{1}\left(\Psi^{1}\left(x, v^{1}\right)\right) \operatorname{div} \operatorname{grad} \Psi\left(x, v^{1}\right)$

onde $\operatorname{grad} \Psi^{1}=\operatorname{col}\left(\frac{\partial \Psi^{1}}{\partial x_{1}}, \ldots, \frac{\partial \Psi^{1}}{\partial x_{n}}\right)$, sendo $\frac{\partial \Psi^{1}}{\partial x_{i}}$ a derivada com respeito a $x_{i}$, estando fixada a variável $\theta$ e calculada em $\left(x, v^{1}(t, x)\right) ; \operatorname{grad} \Psi^{1}\left(x, v^{1}\right)=$ $\operatorname{col}\left(\frac{d \Psi^{1}}{d x_{1}}, \ldots, \frac{d \Psi^{1}}{d x_{n}}\right)$. Definindo as funções reais

$$
\begin{aligned}
d_{1}^{1}(x, \theta) & =D^{1}\left(\Psi^{1}(x, \theta)\right) \\
a_{1}(x, \theta) & =\frac{D_{\theta}^{1}\left(\Psi_{\theta}^{1}\right)^{2}+D^{1} \Psi_{\theta \theta}^{1}}{\Psi_{\theta}^{1}} \\
\alpha_{1}(x, \theta) & =\frac{2 D_{\theta}^{1} \Psi_{\theta}^{1} \operatorname{grad} \Psi^{1}+2 D^{1} \operatorname{grad} \Psi_{\theta}^{1}}{\Psi_{\theta}^{1}} \\
b_{1}(x, \theta) & =\frac{D_{\theta}^{1}\left|\operatorname{grad} \Psi^{1}\right|^{2}+D^{1} \Delta \Psi^{1}+F^{1}}{\Psi_{\theta}^{1}}
\end{aligned}
$$

obtém-se, usando (3.22)-(3.26),

$$
\frac{\partial v^{1}}{\partial t}=d_{1}^{1} \Delta v^{1}+a_{1}\left|\operatorname{grad} v^{1}\right|^{2}+\alpha_{1} \cdot \operatorname{grad} v^{1}+b_{1}
$$


em $(t, x) \in\left(t_{0}, t_{1}\right] \times \Omega$. Note que temos que garantir a priori que $\left|v^{1}\right| \leq B+1$ para que $\Psi^{1}>0$, conforme segue de (3.18).

Analogamente, aplicando a regra da cadeia antes de fazer a substituição de $u^{j}(t, x)=\Psi^{j}\left(x, v^{1}(t, x), v^{j}(t, x)\right)$ em (3.1), com $j \neq 1$, tem-se

$$
\frac{\partial \Psi^{j}}{\partial t}=\Psi_{\phi}^{j} \frac{\partial v^{1}}{\partial t}+\Psi_{\theta}^{j} \frac{\partial v^{j}}{\partial t}
$$

$\operatorname{grad} \Psi^{j}\left(x, v^{1}, v^{j}\right)=\operatorname{grad} \Psi^{j}+\Psi_{\phi}^{j} \operatorname{grad} v^{1}+\Psi_{\theta}^{j} \operatorname{grad} v^{j}$

$\operatorname{grad} D^{j}\left(\Psi^{1}\left(x, v^{1}\right)\right)=D_{\theta}^{j} \operatorname{grad} \Psi^{1}+D_{\theta}^{j} \Psi_{\theta}^{1} \operatorname{grad} v^{1}$

$$
\operatorname{div} \operatorname{grad} \Psi^{j}\left(x, v^{1}, v^{j}\right)=\Delta \Psi^{j}+\operatorname{grad} \Psi_{\phi}^{j} \cdot \operatorname{grad} v^{1}+
$$

$$
+\operatorname{grad} \Psi_{\theta}^{j} \cdot \operatorname{grad} v^{j}+\operatorname{grad} \Psi_{\phi}^{j} \cdot \operatorname{grad} v^{1}+
$$

$+\Psi_{\phi \phi}^{j}\left|\operatorname{grad} v^{1}\right|^{2}+\Psi_{\phi \theta}^{j} \operatorname{grad} v^{1} \cdot \operatorname{grad} v^{j}+\Psi_{\phi}^{j} \Delta v^{1}$

$+\operatorname{grad} \Psi_{\theta}^{j} \cdot \operatorname{grad} v^{j}+\Psi_{\theta \phi}^{j} \operatorname{grad} v^{1} \cdot \operatorname{grad} v^{j}+$

$+\Psi_{\theta \theta}^{j}\left|\operatorname{grad} v^{j}\right|^{2}+\Psi_{\theta}^{j} \Delta v^{j}$

$\operatorname{div} D^{j}\left(\Psi^{1}\left(x, v^{1}\right)\right) \operatorname{grad} \Psi^{j}\left(x, v^{1}, v^{j}\right)=\operatorname{grad} D^{j}\left(\Psi\left(x, v^{1}\right)\right) \cdot \operatorname{grad} \Psi^{j}\left(x, v^{1}, v^{j}\right)+$ $D^{j}\left(\Psi^{1}\left(x, v^{1}\right)\right) \operatorname{div} \operatorname{grad} \Psi^{j}\left(x, v^{1}, v^{j}\right)$.

Definindo as funções reais, com $j \neq 1$,

$$
\begin{aligned}
d_{1}^{j}(x, \phi, \theta) & =-\frac{\Psi_{\phi}^{j}}{\Psi_{\theta}^{j}} d_{1}^{1} \\
d_{j}^{j}(x, \phi, \theta)= & \frac{D^{j}\left(\Psi^{1}\right)}{\Psi_{\theta}^{j}} \\
a_{j}(x, \phi, \theta)= & \frac{D^{j} \Psi_{\phi \phi}+D_{\theta}^{j} \Psi_{\phi}^{j} \Psi_{\theta}^{1} \Psi_{\phi}^{j} a_{1}}{\Psi_{\theta}^{j}} \\
b_{j}(x, \phi, \theta)= & \frac{D^{j} \Psi_{\theta \theta}^{j}}{\Psi_{\theta}^{j}} \\
c_{j}(x, \phi, \theta)= & \frac{D_{\theta}^{j}\left(\Psi_{\theta}^{j}\right)^{2}+2 D^{j} \Psi_{\theta \phi}^{j}}{\Psi_{\theta}^{j}} \\
\alpha_{\mathbf{j}}(x, \phi, \theta)= & \frac{1}{\Psi_{\theta}^{j}}\left(D_{\theta}^{j} \Psi_{\phi}^{j} \operatorname{grad} \Psi^{1}+D_{\theta}^{j} \Psi_{1}^{\theta} \operatorname{grad} \Psi^{j}\right. \\
& \left.+2 D^{j} \operatorname{grad} \Psi_{\phi}^{j}-\Psi_{\phi}^{j} \alpha_{1}\right) \\
\beta_{\mathbf{j}}(x, \phi, \theta)= & \frac{D_{\theta}^{j} \Psi_{\theta}^{j} \operatorname{grad} \Psi^{1}+2 D^{j} \operatorname{grad} \Psi_{\theta}^{j}}{\Psi_{\theta}^{j}}
\end{aligned}
$$




$$
\begin{aligned}
\gamma_{\mathbf{j}}(\mathrm{x}, \phi, \theta)= & \frac{D_{\theta}^{j} \Psi_{\theta}^{j} \operatorname{grad} \Psi^{j}+2 D^{j} \operatorname{grad} \Psi_{\theta}^{j}}{\Psi_{\theta}^{j}} \\
e_{j}(x, \phi, \theta)= & \frac{1}{\Psi_{\theta}^{j}}\left(D_{\theta}^{j} \operatorname{grad} \Psi^{j} \cdot \operatorname{grad} \Psi^{j}+\Delta \Psi^{j}\right. \\
& \left.F^{j}-\Psi_{\phi}^{j} b_{1}\right)
\end{aligned}
$$

obtém-se, usando (3.33)-(3.37),

$$
\begin{aligned}
\frac{\partial v^{j}}{\partial t}= & d_{1}^{j} \Delta v^{1}+d_{j}^{j} \Delta v^{j}+a_{j}\left|\operatorname{grad} v^{1}\right|^{2}+ \\
& +b_{j}\left|\operatorname{grad} v^{j}\right|^{2}+c_{j} \operatorname{grad} v^{1} \cdot \operatorname{grad} v^{j}+\beta_{\mathbf{j}} \cdot \operatorname{grad} v^{1} \\
& +\gamma_{\mathbf{j}} \cdot \operatorname{grad} v^{j}+e_{j} \quad \\
& \quad j=1, \ldots, m,
\end{aligned}
$$

onde incluímos aqui também a equação (3.32) definindo os coeficientes não presentes em (3.32) como zero. Devido as condições impostas a $g^{j}$ e $D^{j}$ na seção 3.1 .1 , podemos dizer algo comum a todas as funções definidas acima relativamente à regularidade.

Proposição 3.1.5 As funções definidas por (3.27)-(3.30) são de classe $C^{0, \epsilon}$ no domínio $(x, \theta) \in \bar{\Omega} \times\{\theta \in \mathbb{R} \| \theta \mid \leq B+1\}$ e as funções definidas por (3.38)-(3.46) são de classe $C^{0, \epsilon}$ no domínio $(x, \phi, \theta) \in \bar{\Omega} \times\{\phi \in \mathbb{R}|| \phi \mid \leq$ $B+1\} \times\{\theta \in \mathbb{R}|| \theta \mid \leq B+1\}$.

Prova. Basta notar que as derivadas mais altas de $\Psi^{j}$ e $D^{j}$ que aparecem nestas definições são, respectivamente, de ordem 2 e $1, \Psi_{\theta}^{j} \neq 0$ nos domínios acima devido a (3.18), $\Psi^{j}$ é de classe $C^{2, \epsilon}$ devido a condição 4 e a sua definição e $D^{j}$ é de classe $C^{1, \epsilon}$ devido a condição 2 .

Completamos a descrição do problema, definindo as novas condições iniciais $v_{0}^{j}(x)$ também implicitamente por

$$
\left\{\begin{array}{c}
\Psi^{1}\left(x, v_{0}^{1}(x)\right)=u_{0}^{1}(x) \\
\Psi^{j}\left(x, v_{0}^{1}(x), v_{0}^{j}(x)\right)=u_{0}^{j}(x) .
\end{array}\right.
$$

Teorema 3.1.6 Os problemas (3.1)-(3.2)-(3.3) e (3.47)-(3.21)-(3.48) que chamamos, respectivamente, de $(P)$ e $(\hat{P})$ são equivalentes nos sentidos 
1. Se $\mathbf{u}(t, x)=\operatorname{col}\left(u^{1}(t, x), \ldots, u^{m}(t, x)\right)$ é solução clássica de $(P)$ tal que $\left|u^{j}(t, x)\right| \leq B$, qualquer $j$, em $\left[t_{0}, t_{1}\right] \times \bar{\Omega}$ então $\mathrm{v}$ é solução clássica do problema $(\hat{P})$ tal que $\left|v^{j}(t, x)\right| \leq B+1$ no mesmo dominio, qualquer $j$

2. Inversamente se $\mathbf{v}$ é solução clássica de $(\hat{P})$ tal que $\left|v^{j}(t, x)\right| \leq B+1$ em $\left[t_{0}, t_{1}\right] \times \bar{\Omega}$, qualquer $j$, então u é solução clássica de $(P)$ tal que $|u(t, x)| \leq B+2$ no mesmo domínio, qualquer $j$.

Prova. 1. Seja $\Psi_{(x)}^{1}(\theta)=\Psi^{1}(x, \theta)$. Temos que $[-B-1, B+1] \ni \theta \rightarrow$ $\Psi_{(x)}^{1}(\theta)$ é estritamente crescente devido a (3.18) e por (3.17)

$$
\Psi_{(x)}^{1}(-B-1) \leq-B \text { e } \Psi_{(x)}^{1}(B+1) \geq B,
$$

para todo $x \in \bar{\Omega}$. Resulta que a equação (3.19) (ou (3.48), $j=1$, se $t=t_{0}$ ) define implicitamente uma única $v^{1}, v^{1}(t, x)=\left(\Psi_{(x)}^{1}\right)^{-1}(u(t, x))$, sendo a inversa tomada em relação á variável $\theta$ e $\left|v^{1}(t, x)\right| \leq B+1$. Do teorema da função implícita, segue que $v^{1}$ tem a mesma regularidade de $u^{1}$ e o cálculo precedente mostra que $v^{1}$ satisfaz a equações (3.47),(3.21),(3.48), com $j=1$. Conhecida $v^{1}(t, x)$, definimos a função, $\operatorname{com} j \neq 1$,

$$
[-B-1, B+1] \ni \theta \rightarrow \Psi_{\left(x, v^{1}(t, x)\right.}^{j}(\theta),
$$

onde $\Psi_{\left(x, v^{1}(t, x)\right)}^{j}(\theta)=\Psi^{j}\left(x, v^{1}(t, x), \theta\right)$ cuja imagem também contém $[-B, B] \mathrm{e}$ é estritamente crescente. Segue igualmente que a equação (3.20) define uma única $v^{j}, v^{j}(t, x)=\left(\Psi_{\left(x, v^{1}(t, x)\right)}^{j}\right)^{-1}\left(u^{j}(t, x)\right)$, que satisfaz, devido ao cálculo precedente, a equação $j$ de $(3.47),(3.21),(3.48)$. A prova de 2 é direta e vai ser omitida. 


\section{Capítulo 4}

\section{Resultados de Sobolevskiū e aplicação}

Muitos resultados tratados no presente capítulo exigem grande capacidade de abstração do leitor. Na maioria das vezes, vamos apenas enunciá-los o que certamente não é suficiente para se enxergar a grande quantidade de idéias que contém. Por exemplo, não parece nada evidente que o teorema de Sobolevskiü(seção 4.1.1) tenha muita teoria de semigrupos de operadares lineares atrás de si. As referências (e referências achadas aí) que damos podem suprir tal deficiência.

Vamos usar o conteúdo da primeira seção do presente capítulo na seção 4.2 para provar existência e unicidade de solução local no tempo para o problema (3.47)-(3.21)-(3.48) (4.2.2) a qual é, graças também a resultados de regularidade para equações elípticas [2], a sua própria solução clássica. Daí, usando o teorema 3.1.6 prova-se automaticamente a existência e unicidade de solução clássica para o problema (3.1)-(3.2)-(3.3).

O nosso plano é o seguinte.

Começamos com o teorema de existência e unicidade de Soboleviskï para equação parabólica não-linear que está demonstrado no texto do autor homônimo [8] e também em Friedmann [11]. O enunciado daqui é um pouco diferente do original porque usamos a notação de espaço de potência de operador setorial do texto de Henry[5], p. 24, onde também podem ser achadas a definição e propriedades destes espaços.

O teorema 4.1.2, um resultado de regularização para equação parabólica não-linear, é enunciado e demonstrado somente porque não achamos uma referência explícita para o mesmo. Sua demonstração é uma simples reunião 
de resultados de [8], seguindo o resultado análogo para equação semilinear de [5]. Em seguida, fazemos um rápido estudo a respeito da dependência da solução abstrata da equação parabólica não-linear em relação ao dado inicial que exceto pela forma reproduz os resultados de Amann [6] que por sua vez são baseados nos de Henry [5].

Finalmente, provamos a existência e unicidade de solução local no tempo para o problema (3.1)-(3.2)-(3.3).

\subsection{Equação parabólica não-linear}

Nesta seção, enunciamos o teorema de existência e unicidade de solução abstrata local no tempo (teorema 4.1.1) para equação parabólica não-linear ([8],p.48. [12], p.169), usando uma notação que remete a Henry [5]. Provamos um resultado de regularização 4.1 .2 para esta solução análogo ao de Henry [5](teorema 3.5.2, p.71). O teorema 4.1 .3 é dedicado ao estudo da dependência da solução com relação ao dado inicial.

\subsubsection{Teorema de existêcia e unicidade}

Consideremos o problema de evolução no espaço de Banach $X$

$$
\begin{aligned}
\frac{d u}{d t}+A(t, u) u & =f(t, u), \text { em }\left(t_{0}, t_{1}\right] \\
u\left(t_{0}\right) & =u_{0}
\end{aligned}
$$

onde (4.1) é, em geral, uma equação não-linear em $u$, tal que as condições abaixo são satisfeitas.

1. O operador $A_{0}=A\left(t_{0}, u_{0}\right)$ é fechado com domínio $D\left(A_{0}\right)$ denso em $X$ $e$

$$
\left\|\left(\lambda-A_{0}\right)^{-1}\right\|_{\mathcal{L}(X)} \leq \frac{C}{1+|\lambda|} \text { se } \operatorname{Re} \lambda \leq 0
$$

para uma constante $C$ não dependendo de $\lambda$.

A condição acima nos permite considerar os espaços de potência $X_{0}^{\nu}$, com $\nu \geq 0$, isto é, o domínio das $\nu$-potências de $A_{0}$ ([5], p. 24), munido da norma \|\|$_{\nu}$, tal que se $x \in X_{0}^{\nu}$, tem-se $\|x\|_{\nu}=\left\|A_{0}^{\nu} x\right\|_{X}$. Denotemos entào por $B\left(R, X_{0}^{\nu}\right)$ a bola de raio $R>0$, centro 0 , no espaço de Banach $X_{0}^{\nu}$. 
2. O domínio do operador $A(t, v), D(A(t, v))=D\left(A_{0}\right)$ se $(t, v) \in\left[t_{0}, t_{1}\right] \times$ $B\left(R, X_{0}^{\alpha}\right)$ para algum $R>0$ e $\alpha \in[0,1)$ e para todo $(t, v) \in\left[t_{0}, t_{1}\right] \times$ $B\left(R, X_{0}^{\alpha}\right)$ tem-se

$$
\left\|(\lambda-A(t, v))^{-1}\right\|_{\mathcal{L}(X)} \leq \frac{C}{1+|\lambda|} \text { se } \operatorname{Re} \lambda \leq 0,
$$

para uma constante $C$ não dependendo de $t, v, e \lambda$.

Para todo $t, \tau \in\left[t_{0}, t_{1}\right]$ e $v, w \in B\left(R, X_{0}^{\alpha}\right)$ tem-se

$$
\left\|(A(t, v)-A(\tau, w)) A^{-1}(\tau, w)\right\|_{\mathcal{L}(X)} \leq C(R)\left(|t-\tau|^{\sigma}+\|v-w\|_{\alpha}\right),
$$

para algum $\sigma, \sigma \in(0,1]$.

3. Para todo $t, \tau \in\left[t_{0}, t_{1}\right]$ e $v, w \in B\left(R, X_{0}^{\alpha}\right)$ tem-se

$$
\|f(t, v)-f(\tau, w)\|_{X} \leq C(R)\left(|t-\tau|^{\sigma}+\|v-w\|_{\alpha}\right)
$$

para algum $\sigma, \sigma \in(0,1]$.

4. Assuma que $u_{0} \in X_{0}^{\beta}$ para algum $\beta \in(\alpha, 1]$ e que $u_{0} \in B\left(R, X_{0}^{\alpha}\right)$.

A desigualdade (4.4) implica que para cada $t, v$, o operador $A(t, v)$ é o gerador de um semigrupo analítico ([11], p.101). Por este motivo, a equação (4.1) é chamada de parabólica.

A seguir, damos o teorema que constitui o principal resultado de Sobolevskili que vamos usar aqui.

Teorema 4.1.1 Suponhamos que sejam verificadas as condições 1 a 4. Então existe um número $t^{*}, t_{0}<t^{*} \leq t_{1}$ tal que há uma única solução $u(t)$ de (4.1) que satisfaz (4.2), com $u(t) \in D\left(A_{0}\right)$ se $t \in\left(t_{0}, t^{*}\right]$, contínua em $\left[t_{0}, t^{*}\right]$, continuamente diferenciável em $\left(t_{0}, t^{*}\right]$.

Prova. Sobolevskiǐ [8], p.48 e Friedman [11], p.169.

\subsubsection{Regularização}

Podemos estabelecer o seguinte resultado de regularização para a solução de (4.1)-(4.2) dada pelo teorema 4.1.1.

Teorema 4.1.2 A solução de (4.1)-(4.2) dada pelo teorema 4.1.1 é tal que 
1. $\left[t_{0}, t^{*}\right] \ni t \rightarrow u(t) \in B\left(R, X_{0}^{\alpha}\right)$ é uniformemente Hölder contínua com expoente $\eta, 0<\eta<\beta-\alpha$;

2. $\left[t_{0}+\delta, t^{*}\right] \ni t \rightarrow \frac{d u}{d t}(t) \in X_{0}^{\gamma}$ é uniformemente Hölder contínua, qualquer $0<\delta<t^{*}-t_{0}$, para todo $0 \leq \gamma<\nu<\min (\sigma, \beta-\alpha)=\mu$ e tem-se, para qualquer $\epsilon>0$ e $\forall t_{0}<t<t+h<t^{*}$

$$
\left\|\frac{d u}{d t}(t+h)-\frac{d u}{d t}(t)\right\|_{\gamma} \leq C(\alpha, \gamma, \nu, \epsilon)\left(t-t_{0}\right)^{\beta-\gamma-1-\epsilon} h^{\nu-\gamma} .
$$

Prova. Fazendo a mudança de notação, a primeira afirmação é uma consequência imediata da prova do teorema 4.1.1 (Friedman [11], p.169).

Provemos a segunda. Sem perda de generalidade podemos assumir $t_{0}=0$. De fato, suponha que tenhamos provado (4.7) neste caso e consideremos o problema de provar (4.7) quando $t_{0} \neq 0$ e as condições 1 a 4 estão satisfeitas para os termos de (4.1)-(4.2). Fazendo a mudança de variável $s=t+t_{0}$ e definindo

$$
\begin{aligned}
v(s) & =u\left(s+t_{0}\right) \\
A_{1}(s, v(s)) & =A\left(s+t_{0}, v(s)\right) \\
f_{1}(s, v(s)) & =f\left(s+t_{0}, v(s)\right)
\end{aligned}
$$

como $v^{\prime}(s)=u^{\prime}\left(s+t_{0}\right)$, que obtém-se derivando ambos os membros de (4.8) com relação a $s$, resulta tomando o valor em $s+t_{0}$ na (4.1) e em $s=0$ na (4.2)

$$
\begin{aligned}
\frac{d v}{d s}(s)+A_{1}(s, v(s)) v(s) & =f_{1}(s, v(s)), t \in\left(0, t^{*}-t_{0}\right] \\
v(0) & =u\left(t_{0}\right)
\end{aligned}
$$

Obviamente as condições 1 a 4 valem também para os termos da equação em $v$ acima. Como assumimos que já foi provado que

$$
\left\|\frac{d v}{d t}(t+h)-\frac{d v}{d t}(t)\right\|_{\gamma} \leq C(\alpha, \gamma, \nu, \epsilon) t^{\beta-\gamma-1-\epsilon} h^{\nu-\gamma},
$$

revertendo a mudança de variável de $v$ para $u$, obtém-se (4.7).

Consideremos então somente o caso $t_{0}=0$. Sendo $u(t)$ a solução de (4.1)-(4.2), conforme o teorema 4.1.1, denotemos $A(t)=A(t, u(t)), f(t)=$ 
$f(t, u(t))$ e $U(t, \tau)=U_{u(.)}(t, \tau)$, o operador de evolução associado a $\frac{d x}{d t}+$ $A(t, u(t)) x=0$. Pela primeira afirmação e por (4.6) e (4.5) segue que $\left[t_{0}, t^{*}\right] \ni$ $t \rightarrow f(t)$ e $\left[t_{0}, t^{*}\right] \ni t \rightarrow A(t) A_{0}^{-1}$ são unifomemente Hölder contínuas com expoente $\mu$. Sabemos que para qualquer $t \in\left[0, t^{*}\right]([11]$, p.109 (teorema3.2)),

$$
u(t)=U(t, 0) u_{0}+\int_{0}^{t} U(t, s) f(s) d s .
$$

Daí por (4.1), com $t>0$

$$
\begin{aligned}
\frac{d u}{d t} & =-A(t) U(t, 0) u_{0}-A(t) \int_{t}^{t} U(t, s) f(s) d s+f(t) \\
& =-A(t) U(t .0) u_{0}+\frac{d}{d t} \int_{0}^{t} U(t, s) f(s) d s
\end{aligned}
$$

onde a última igualdade está provada em [11], p.129 e [8], p.32.

Usando lema 14.3 de [11], p.162 e lema 14.5 de [11], p.164 e que $u_{0} \in X_{0}^{\beta}$, o que implica $u_{0}=A_{0}^{-\beta} A_{0}^{\beta} u_{0}$, obtém-se

$$
\left\|\frac{d u}{d t}(t+h)-\frac{d u}{d t}(t)\right\|_{\gamma} \leq C(\sigma, \eta, \beta, \gamma, \epsilon) t^{\beta-\nu-1-\epsilon} h^{\nu-\gamma}+C(\gamma, \nu, \epsilon) t^{-\nu-\epsilon} h^{\nu-\gamma} .
$$

\subsubsection{Dependência em relação ao dado inicial}

Estabelecemos aqui que a solução do problema parabólico não-linear em questão tem dependência Lipschitz contínua em relação ao dado inicial. Dependendo da norma usada, proveniente dos espaços de potência $X_{0}^{\mu}$, com $0 \leq \mu<1$, para medir a variação da solução com relação à variação do dado inicial medida no espaço $X_{0}^{\beta}$, esta regularidade pode ser perdida quando $t \rightarrow t_{0}$.

Teorema 4.1.3 Seja solução de (4.1)-(4.2) dada pelo teorema 4.1.1 denotada por $u\left(t ; u_{0}\right)$, onde, como sabemos, $u_{0} \in X_{0}^{\beta} \cap B\left(R, X_{0}^{\alpha}\right)$. Denotando também analogamente $v\left(t ; v_{0}\right)$, a solução com condição inicial $v_{0} \in$ $X_{0}^{\beta} \cap B\left(R, X_{0}^{\alpha}\right)$, tem-se 
1. para $t \in\left[t_{0}, t^{*}\right]{ }^{1}$

$$
\left\|u\left(t ; u_{0}\right)-v\left(t ; v_{0}\right)\right\|_{\alpha} \leq C\left\|u_{0}-v_{0}\right\|_{\beta}
$$

2. para $t \in\left[t_{0}, t^{*}\right]$ e $\alpha<\mu<\beta$,

$$
\left\|u\left(t ; u_{0}\right)-v\left(t ; v_{0}\right)\right\|_{\mu} \leq C\left\|u_{0}-v_{0}\right\|_{\beta} ;
$$

3. para $t \in\left(t_{0}, t^{*}\right]$ e $\beta \leq \mu<1$,

$$
\left\|u\left(t ; u_{0}\right)-v\left(t ; v_{0}\right)\right\|_{\mu} \leq C t^{\beta-\mu-\epsilon}\left\|u_{0}-v_{0}\right\|_{\beta},
$$

qualquer $\epsilon>0$.

Prova. Sem perda de generalidade podemos assumir $t_{0}=0$.

Usando o mesmo argumento usado para provar a relação (16.24) de [11], p.173, obtém-se

$$
\begin{aligned}
u(t)-v(t)= & U_{u}(t, 0)\left(u_{0}-v_{0}\right)+\int_{0}^{t} U_{u}(t, s)[(A(s, v(s)) \\
& -A(s, u(s))) v(s)+f(s, u(s))-f(s, v(s))] d s,
\end{aligned}
$$

onde $U_{u}(t, s)$ é o operador de evolução associado a $\frac{d w}{d t}+A(t, u(t)) w(t)=0$.

Denotemos $A(t, u(t))=A_{u}(t)$ e $A(t, v(t))=A_{v}(t)$.

Podemos escrever, para $0 \leq \mu<1$,

$$
\begin{aligned}
A_{0}^{\mu}(u(t)-v(t))= & A_{0}^{\mu} A_{u}^{-\mu-\epsilon_{1}}(t) A_{u}^{\mu+\epsilon_{1}}(t) U_{u}(t, 0) A_{0}^{-\beta} A_{0}^{\beta}\left(u_{0}-v_{0}\right) \\
& +\int_{0}^{t} A_{0}^{\mu} A_{u}^{-\mu-\epsilon_{2}}(t) A_{u}^{\mu+\epsilon_{2}}(t) U_{u}(t, s)\left[\left(A_{v}(s)-A_{u}(s)\right) v(s)\right. \\
& +f(s, u(s))-f(s, v(s))] d s .
\end{aligned}
$$

Provemos o caso 1 . Tomemos $\mu=\alpha, \epsilon_{1} \geq \beta-\alpha$. Usando a relação

$$
\left\|A_{0} v(s)\right\| \leq c s^{\beta-1}
$$

\footnotetext{
${ }^{1}$ Estamos tomando $t^{*}=\min \left\{t^{*}\left(u_{0}\right), t^{*}\left(v_{0}\right)\right\}$.
} 
provada em [11], p.173 ou [8], p.51, aplicando as relações (14.12) e (14.14) de Friedman ([11],p.160 e p.161) e (4.5), (4.6) tem-se

$$
\begin{aligned}
\|u(t)-v(t)\|_{\alpha} & \leq C t^{\beta-\alpha-\epsilon_{1}}\left\|u_{0}-v_{0}\right\|_{\beta}+C \int_{0}^{t}(t-s)^{-\alpha-\epsilon_{2}}\left(s^{\beta-1}+1\right)\|u(s)-v(s)\|_{\alpha} d s \\
& \leq C t^{\beta-\alpha-\epsilon_{1}}\left\|u_{0}-v_{0}\right\|_{\beta}+C \int_{0}^{t}(t-s)^{-\alpha-\epsilon_{2}} s^{\beta-1}\|u(s)-v(s)\|_{\alpha} d s,
\end{aligned}
$$

onde usamos também que $1=s^{1-\beta} s^{\beta-1} \leq C s^{\beta-1}$. Escolhendo $\epsilon_{2}>0$ tal que

$$
1-\alpha-\epsilon_{2}+\beta-1>0,
$$

isto é, $\epsilon_{2}<\beta-\alpha$ e também escolhendo $\epsilon_{1} \geq \beta-\alpha$ tal que

$$
\beta-\alpha-\epsilon_{1}+\beta>0,
$$

isto é, $\epsilon_{1}<2 \beta-\alpha$ podemos aplicar a (4.24) o resultado de Henry[5], seção 7.1 , exercício 3 , para obter

$$
\|u(t)-v(t)\|_{\alpha} \leq C t^{\beta-\alpha-\epsilon_{1}}\left\|u_{0}-v_{0}\right\|_{\beta} .
$$

Finalmente, tomando $\epsilon_{1}=\beta-\alpha$, tem-se (4.18).

Tratemos dos demais casos.

Voltando a (4.22), tomando $\mu \geq \alpha, \epsilon_{1} \geq \beta-\mu$ e $\epsilon_{1}>0$, aplicando os lemas de Friedman como antes e usando a relação (4.25), chega-se a

$$
\|u(t)-v(t)\|_{\mu} \leq C\left(t^{\beta-\mu-\epsilon_{1}}+\int_{0}^{t}(t-s)^{-\alpha-\epsilon_{2}}\left(s^{\beta-1}+1\right) d s\right)\left\|u_{0}-v_{0}\right\|_{\beta},
$$

e notemos que se $0<\epsilon_{2}<\beta-\alpha$,

$$
\begin{aligned}
\int_{0}^{t}(t-s)^{-\alpha-\epsilon_{2}}\left(s^{\beta-1}+1\right) d s= & \int_{0}^{t / 2}+\int_{t / 2}^{t} \leq C\left(\left(\frac{t}{2}\right)^{-\alpha-\epsilon_{2}} \frac{t^{\beta}}{2^{\beta}}+\right. \\
& \left(\frac{t}{2}\right)^{\beta-1}\left(\frac{t}{2}\right)^{1-\alpha-\epsilon_{2}} \leq C^{*}
\end{aligned}
$$

Primeiramente, se $a<\mu<\beta$, tomando $\epsilon_{1}=\beta-\mu$ e usando (4.26), obtém-se (4.19). Quando $1>\mu \geq \beta$, escolhemos $\epsilon_{1}=\epsilon$, qualquer $\epsilon>0$ sufientemente pequeno para obtermos (4.20). 


\subsection{Aplicação}

Vamos aplicar os resultados precedentes na prova de existência e unicidade de solução local no tempo para o problema (3.47)-(3.21)-(3.48).

Definamos com $v(x)=\operatorname{col}\left(v^{1}(x), \ldots, v^{m}(x)\right)$ e $x \in \bar{\Omega}$

$$
A(v)(x)=\left(\begin{array}{cccc}
-d_{1}^{1}\left(x, v^{1}\right) \Delta+k_{0} & \cdots & 0 & 0 \\
-d_{1}^{2}\left(x, v^{1}\right) \Delta & -d_{2}^{2}\left(x, v^{1}, v^{2}\right) \Delta+k_{0} & \ldots & 0 \\
\vdots & \vdots & \vdots & \vdots \\
-d_{1}^{m}\left(x, v^{1}\right) \Delta & \cdots & 0 & -d_{m}^{m}\left(x, v^{1}, v^{m}\right) \Delta+k_{0}
\end{array}\right)
$$

onde $k_{0}>0$ é uma constante suficientemente grande. E também definamos

$$
f(v)(x)=\left(\begin{array}{c}
a_{1}\left|\operatorname{grad} v^{1}\right|^{2}+\alpha_{1} \cdot \operatorname{grad} v^{1}+b_{1}+k_{0} v^{1} \\
\vdots \\
a_{m}\left|\operatorname{grad} v^{1}\right|^{2}+b_{j}\left|\operatorname{grad} v^{j}\right|^{2}+\ldots+e_{j}+k_{0} v^{m}
\end{array}\right)
$$

onde os coeficientes com índice 1 são calculados em $\left(x, v^{1}\right)$ e os coeficientes com índice $j \neq 1$ são calculados em $\left(x, v^{1}, v^{j}\right)$ e ... é preenchido pelos mesmos termos entre $b_{j}\left|\operatorname{grad} v^{j}\right|^{2}$ e $e_{j}$ como na (3.47).

Segue que a equação (3.47) e a condição inicial (3.48) podem ser escritas como

$$
\begin{array}{r}
\frac{d v}{d t}(t)+A(v) v=f(v), t \in\left(t_{0}, t_{1}\right] \\
v(0)=v_{0}
\end{array}
$$

onde $\frac{d v}{d t}(t)(x)=\frac{\partial v}{\partial t}(t, x)$ e as igualdades são válida em todo $x \in \Omega$.

Consideremos que o domínio de $A(v)$ para qualquer $v$ é dado por

$$
D(A(v))=W_{N}^{2, p}(\Omega) \times \ldots \times W_{N}^{2, p}(\Omega),
$$

$m$ vezes, onde $W_{N}^{2, p}(\Omega)$ denota o subespaço do espaço de Sobolev $W^{2, p}$,

$$
W_{N}^{2, p}(\Omega)=\left\{\phi \in W^{2, p}(\Omega) \mid \frac{\partial \phi}{\partial N}=0\right\} .
$$

Vamos assumir que $p \in(n, \infty)$ e então $W^{2, p}(\Omega) \subset C^{1, \epsilon}(\bar{\Omega})$, para qualquer $\epsilon \in[0,1-n / p)$, com imersão contínua (Adams [13], teorema 5.4, caso C', 
p.98). Daí segue que a derivada normal ao bordo acima tem a interpretação usual.

Antes de aplicar o teorema 4.1.1, lembremos alguns fatos sobre o problema de valor de bordo

$$
\begin{aligned}
-d(x) \Delta \phi+k_{0} \phi & =h(x), x \in \Omega \\
\frac{\partial \phi}{\partial N}(x) & =0, x \in \partial \Omega .
\end{aligned}
$$

onde $k_{0}$ é escolhido suficientemente grande, $d(x)>c_{0}>0$, para todo $x \in \Omega$ e $d$ é uma função de classe $C^{0, \epsilon}(\bar{\Omega})$ para algum $\epsilon \in(0,1]$.

Como caso particular do exemplo dado em Friedman [12]. p.101, resulta que o problema acima define um operador $-d(x) \Delta+k_{0}$ fechado com domínio $W_{N}^{2, p}$ cujo conjunto resovente contém o setor

$$
S(k, \varphi)=\{z \in C \mid z \neq k ; \arg (z-k)>\pi / 2-\varphi\}
$$

para algum $k \in \mathbb{R}, \varphi \in(0, \pi / 2)$ e tem-se

$$
\left\|\left(\lambda-\left(-d(x) \Delta+k_{0}\right)\right)^{-1}\right\|_{\mathcal{L}\left(L_{p}\right)} \leq \frac{M}{|\lambda|} \text { se } \lambda \in S^{\prime}(k, \nu) .
$$

Então, tomando $k_{0}$ conveniente, podemos supor que $k>0 \mathrm{e}$

$$
\left\|\left(\lambda-\left(-d(x) \Delta+k_{0}\right)\right)^{-1}\right\|_{\mathcal{L}\left(L_{p}\right)} \leq \frac{C}{|\lambda|+1} \text { se } \operatorname{Re} \lambda \leq 0 .
$$

Também tem-se uma estimativa do tipo de Schauder para o problema acima (Friedman [12], teorema 19.1, p.74 e mais explícitamente, Rothe [4], lema 1, p.15),

$$
\|\phi\|_{W^{2, p}} \leq C\left(\left\|\left(-d(x) \Delta+k_{0}\right) \phi\right\|_{L_{p}}+\|\phi\|_{L_{p}}\right)
$$

e daí escrevendo $\phi=\left(-d(x) \Delta+k_{0}\right)\left(-d(x) \Delta+k_{0}\right)^{-1} \phi$, usando que $(-d(x) \Delta+$ $\left.k_{0}\right)^{-1}$ é limitado e (4.34)

$$
\|\phi\|_{W^{2, p}} \leq C\|h\|_{L_{p}}
$$

com uma nova constante $C$.

Observação 4.2.1 A constante $C$ nas estimativas acima depende, em relação a $d(x)$, somente de sua norma em $C^{0}$ (note que estamos supondo que $\left.d(x)>c_{0}>0\right)$, com $c_{0}$ constante). Veja exemplo de Friedman [12], p.101 $\epsilon$ sua condição de regularidade da p.74. 
Estamos em condições de aplicar o teorema 4.1.1.

\section{Etapa 1}

Comecemos com algumas observações preliminares.

Suponha que $v_{0} \in C^{1, \epsilon}(\bar{\Omega})$, para algum $\epsilon \in(0,1]$. Então podemos aplicar os fatos acima para o problema de bordo em $\phi_{j}$

$$
\begin{aligned}
-d_{1}^{j}\left(x, v_{0}^{1}, v_{0}^{j}\right) \Delta \phi_{j}+k_{0} \phi_{j} & =h_{j} \\
\frac{\partial \phi_{j}}{\partial N} & =0
\end{aligned}
$$

Daí

$$
\left\|\left(\lambda-\left(-d_{1}^{j}\left(x, v_{0}^{1}, v_{0}^{j}\right) \Delta+k_{0}\right)\right)^{-1}\right\|_{\mathcal{L}\left(L_{p}\right)} \leq \frac{C}{|\lambda|+1} \text { se } \operatorname{Re} \lambda \leq 0,
$$

e também

$$
\left\|\phi_{j}\right\|_{W^{2, p}} \leq C\left\|h_{j}\right\|_{L_{p}} .
$$

Determinação da matriz $\left(\lambda-A_{0}\right)^{-1}$. Para isto, notemos que dado $\mathbf{h}=$ $\operatorname{col}\left(h_{1}, \ldots, h_{m}\right), \operatorname{com} h_{j} \in L_{p}$,

$$
\left(\lambda-A_{0}\right) \phi=\mathrm{h}
$$

é equivalente a

$$
\begin{aligned}
\left(\lambda-\left(-d_{1}^{1} \Delta+k_{0}\right)\right) \phi_{1} & =h_{1} \\
\left(\lambda-\left(-d_{1}^{j} \Delta+k_{0}\right)\right) \phi_{j}+d_{1}^{1} \Delta \phi_{1} & =h_{j} \text { se } j \neq 1
\end{aligned}
$$

tal que $\frac{\partial \phi^{\jmath}}{\partial N}=0$. Resulta que

$$
\begin{aligned}
\phi_{1}= & \left(\lambda-\left(-d_{1}^{1} \Delta+k_{0}\right)\right)^{-1} h_{1} \\
\phi_{j}= & \left(\lambda-\left(-d_{1}^{j} \Delta+k_{0}\right)\right)^{-1} h_{j}- \\
& -d_{1}^{1}\left(\lambda-\left(d_{1}^{j} \Delta+k_{0}\right)\right)^{-1} \Delta\left(\lambda-\left(-d_{1}^{1} \Delta+k_{0}\right)\right)^{-1} h_{1}
\end{aligned}
$$

onde $d_{1}^{1}$ é calculado em $\left(x, v_{0}^{1}\right)$ e $d_{1}^{j}$ é calculado em $\left(x, v_{0}^{1}, v_{0}^{j}\right)$.

Para concluir a prova de (4.3), notemos que

$$
\begin{aligned}
\Delta\left(\lambda-\left(-d_{1}^{1} \Delta+k_{0}\right)\right)^{-1}= & \Delta\left(-d_{1}^{1} \Delta+k_{0}\right)^{-1}\left(-d_{1}^{1} \Delta+k_{0}\right)\left(\lambda-\left(-d_{1}^{1} \Delta+k_{0}\right)\right)^{-1} \\
& \Delta\left(-d_{1}^{1} \Delta+k_{0}\right)^{-1}\left(\lambda\left(\lambda-\left(-d_{1}^{1} \Delta+k_{0}\right)\right)^{-1}-I\right),
\end{aligned}
$$


e como, por (4.43), $\Delta\left(-d_{1}^{1} \Delta+k_{0}\right)^{-1}$ é limitado, aplicando(4.42) tem-se (4.3).

\section{Etapa 2}

Conforme observado após a condição 1 da seção 4.1.1, podemos considerar os espaços potência $X_{0}^{\nu}$. Escolhendo $\alpha \in(1 / 2,1), p>\frac{n}{2 \alpha-1}$, tem-se $X_{0}^{\alpha} \subset$ $C^{1, \epsilon}(\bar{\Omega})$, com imersão contínua, qualquer $\epsilon \in[0,2 \alpha-n / p-1$ ) (Henry [5], teorema 1.6 .1 , p.39). Daí, para qualquer elemento $v$ da bola $B\left(R, X_{0}^{\alpha}\right)$, podemos repetir todo o argumento da etapa 1 para obter (4.4), exceto que $d_{1}^{1}$ é calculado em $\left(x, v^{1}\right)$ e $d_{1}^{j}$ é calculado em $\left(x, v^{1}, v^{j}\right)$. Tem-se unifomidade da estimativa em $B\left(R, X_{0}^{\alpha}\right)$ por ser tal conjunto limitado na norma $C^{0}(\bar{\Omega})$ (mais ainda, na norma $C^{1, \epsilon}$ ) e devido a regularidade de $d_{1}^{1}$ e $d_{1}^{j}$ (i.é., $C^{0, \epsilon}$, proposição 3.1.5, com $\epsilon$ concernente às condições da seção 3.1.1).

Passemos à prova de (4.5). Sejam $v, w \in B\left(R, X_{0}^{\alpha}\right)$. Uma rápida olhada em (4.28) mostra que

$$
A(v)-A(w)
$$

tem elementos da forma $-\left(d_{1}^{j}\left(x, v^{1}, v^{j}\right)-d_{1}^{j}\left(x, w^{1}, w^{j}\right)\right) \Delta$ na primeira coluna $\mathrm{e}-\left(d_{j}^{j}\left(x, v^{1}, v^{j}\right)-d_{j}^{j}\left(x, w^{1}, w^{j}\right)\right) \Delta$ na diagonal principal e demais elementos nulos. Segue que

$$
(A(v)-A(w)) A(v)^{-1} \mathrm{~h}
$$

é o vetor coluna

$$
\left(\begin{array}{c}
-\left(d_{1}^{1}\left(x, v^{1}\right)-d_{1}^{1}\left(x, v^{1}\right)\right) \Delta \phi_{1} \\
\vdots \\
-\left(d_{1}^{j}\left(x, v^{1}, v^{j}\right)-d_{1}^{j}\left(x, v^{1}, v^{j}\right)\right) \Delta \phi_{1}+\ldots \phi_{j} \\
\vdots \\
-\left(d_{1}^{m}\left(x, v^{1}, v^{m}\right)+\right.\text { etc }
\end{array}\right)
$$

onde $\ldots$ substitui $-\left(d_{j}^{j}\left(x, v^{1}, v^{j}\right)-d_{j}^{j}\left(x, w^{1}, w^{j}\right)\right) \Delta ; \phi_{1}$ e $\phi_{j}$ são dados por (4.46) e (4.47) $\operatorname{com} \lambda=0$.

Por (4.43) segue que

$$
\begin{aligned}
\left\|\Delta \phi_{1}\right\|_{L_{p}} & \leq C\left\|h_{1}\right\|_{L_{p}} \\
\left\|\Delta \phi_{j}\right\|_{L_{p}} & \leq C\left(\left\|h_{1}\right\|_{L_{p}}+\left\|h_{j}\right\|_{L_{p}}\right) .
\end{aligned}
$$

Como

$$
\left\|d\left(x, v^{1}, v^{j}\right)-d\left(x, w^{1}, w^{j}\right)\right\|_{C^{0}} \leq C\left(\left\|v^{1}-w^{1}\right\|_{C^{0}}+\left\|v^{j}-w^{j}\right\|_{C^{0}}\right),
$$


devido a proposição 3.1.5 e tomando $\epsilon=1$ nas condições da seção 3.1.1. Daí, como $X_{0}^{\alpha} \subset C^{0}$, com imersão contínua, tem-se (4.5).

\section{Etapa 3}

Provemos (4.6).

Assumindo $\epsilon=1$ nas condições da seção 3.1 .1 e em virtude da proposição 3.1.5, tem-se que todos os coeficientes de (4.29) satisfazem uma desigualdade do tipo de (4.48).

Façamos a prova de (4.6) para um dos termos de (4.29), por exemplo, $a_{1}\left|\operatorname{grad} v^{1}\right|^{2}$. Tem-se

$$
\begin{aligned}
\| a_{1}\left(x, v^{1}\right)\left|\operatorname{grad} v^{1}\right|^{2}- & a_{1}\left(x, w_{1}\right)\left|\operatorname{grad} v^{1}\right|^{2} \|_{L_{p}} \leq \\
\leq \quad & \left\|a_{1}\left(x, v^{1}\right)\left(\left|\operatorname{grad} v^{1}\right|^{2}-\left|\operatorname{grad} w^{1}\right|^{2}\right)\right\|_{L_{p}}+ \\
& +\left\|\left(a_{1}\left(x, v^{1}\right)-a_{1}\left(x, w^{1}\right)\right)\left|\operatorname{grad} w^{1}\right|^{2}\right\|_{L_{p}}
\end{aligned}
$$

e como $X_{0}^{\alpha} \subset C^{1}(\bar{\Omega})$, tem-se

$$
\left\|\operatorname{grad} v^{1}\right\|_{C^{0}},\left\|\operatorname{grad} w^{1}\right\|_{C^{0}} \leq c R
$$

pois $v^{1}, w^{1} \in B\left(R, X_{0}^{\alpha}\right)$. Como $a_{1}$ satisfaz uma desigualdade do tipo de (4.48), tem-se

$$
\begin{aligned}
\| a_{1}\left(x, v^{1}\right)\left|\operatorname{grad} v^{1}\right|^{2}- & a_{1}\left(x, w w^{1}\right)\left|\operatorname{grad} w^{1}\right|^{2} \|_{L_{p}} \leq \\
& \leq C(R)\left\|v^{1}-w^{1}\right\|_{\alpha}
\end{aligned}
$$

onde também foi usado que

$$
\left|\operatorname{grad} v^{1}\right|^{2}-\left|\operatorname{grad} w^{1}\right|^{2} \leq\left(\left|\operatorname{grad} v^{1}\right|+\mid \operatorname{grad} w^{1}\right)\left(\left|\operatorname{grad} v^{1}-\operatorname{grad} w^{1}\right|\right) .
$$

\section{Etapa 4}

Como $X_{0}^{1} \subset X_{0}^{\nu}$, qualquer $\nu \in[0,1]$ (Henry [5], p.25), resulta que se $v_{0}$ satizfaz condição j̆ da seção 3.1.1, então vale condição 4 da seção 4.1.1. Finalmente o $R$ escolhido nas etapas anteriores deve ser tal que $R>\left\|v_{0}\right\|_{\alpha}$.

Então, aplicando o teorema 4.1.1 e teorema 4.1.2, tem-se que o problema (4.30)-(4.31) admite uma solução $v(t)$ em um intervalo $\left[t_{0}, t^{*}\right]$ tal que

$$
\begin{aligned}
& v \in C^{\eta}\left(\left[t_{0}, t^{*}\right], X_{0}^{\alpha}\right) \\
& \frac{d v}{d t} \in C^{\nu-\gamma}\left(\left(t_{0}+\delta, t^{*}\right], X_{0}^{\gamma}\right) .
\end{aligned}
$$


Como $X_{0}^{\alpha} \subset C^{1}(\bar{\Omega})$, com imersão contínua, resulta que $\left[t_{0}, t^{*}\right] \rightarrow v(t), \frac{\partial v}{\partial x_{i}}(t) \in$ $C^{0}(\bar{\Omega})$ são contínuas e portanto

$$
\left[t_{0}, t^{*}\right] \times \bar{\Omega} \rightarrow v(t, x), \frac{\partial v}{\partial x_{i}}(t, x) \in \mathbb{R}^{m}
$$

são contínuas, qualquer $i, 1 \leq i \leq n$. Analogamente, tomando $\gamma \in(0, \beta-\alpha]$ (lembre que $\beta \in(\alpha, 1]$ ) tem-se $X_{0}^{\gamma} \in C^{\epsilon}(\bar{\Omega})$, para algum $\epsilon>0$ (Henry [5], teorema 1.6.1, p.39). Segue que

$$
\left(t_{0}+\delta, t^{*}\right] \times \bar{\Omega} \rightarrow \frac{\partial v}{\partial t}(t, x) \in \mathbb{R}^{m}
$$

é contínua (mais ainda, $C^{\epsilon}(\bar{\Omega})$ para algum $\epsilon>0$ ). Votando a (3.47), tem-se, por exemplo, para $j=1$

$$
\begin{aligned}
-d_{1}^{1}\left(x, v^{1}(x)\right) \Delta v^{1}(x)+k_{0} v^{1}(t, x)= & -\frac{\partial v^{1}}{\partial t}(t, x)+k_{0} v^{1}(t, x)+a_{1}\left|\operatorname{grad} v^{1}\right|^{2}+ \\
& +\alpha_{1} \operatorname{grad} v^{1}+b_{1} \\
\frac{\partial v^{1}}{\partial N}= & 0
\end{aligned}
$$

e aplicando o resultado de regularidade de Ladyzhenskaya [2] (teorema 3.2, p.128), obtemos

$$
\left(t_{0}, t^{*}\right] \times \bar{\Omega} \rightarrow \frac{\partial^{2} v^{j}}{\partial x_{i} x_{k}} \in \mathbb{R}
$$

contínua, qualque $i, k, 1 \leq i, k \leq n$. Podemos repetir o mesmo raciocínio para qualquer $j, 1 \leq j \leq m$.

Então temos uma solução clássica local no tempo para (3.47)-(3.21)-(3.48) e portanto, devido ao teorema 3.1.6, obtém-se também solução clássica local no tempo para o problema (3.1)-(3.2)-(3.3).

Em resumo, temos o segunte proposição.

Proposição 4.2.2 Assuma que sejam satisfeitas condições 2 a 4 da seção $3.1 .1 \operatorname{com} \epsilon=1$ (i.e., as derivada primeira de $D^{j}$, as derivadas segundas de $g^{j} \in$ a $F^{j}$ são Lipschitz contínuas), a condição 1 e a 5. Então o problema (3.1)-(3.2)-(3.3) admite uma única solução clássica local no tempo. 


\subsubsection{Retorno ao modelo físico}

Consideremos o sistema matemático de reação-difusão (SMRD) (2.10), $i=1,2$, e (2.7) com condições de fronteira, respectivamente, (2.12), $i=1,2$, e (2.8) nas variáveis $c_{1}, c_{2}$ e $T(m=3)$ e variáveis independentes $t, x$ e com certas condicões iniciais.

Note que embora muitas das relações do modelo do capitulo 2 sejam dadas explicitamente, como um dos termos da condição de fronteira da equação do calor, nominalmente, a lei de Stefan Boltzman representada por uma função $h_{1}(T)$,

$$
h_{1}(T)=\sigma e\left(T^{4}-T_{0}^{4}\right), \sigma, \text { e constantes, }
$$

que obviamente é $C^{m, 1}$ na variável $T$ em limitados de $\mathbb{R}$, para qualquer $m^{2}$, existem muitas relações em que não conhecemos a expressão analítica para as mesmas. Por exemplo, o segundo termo da condição de fronteira da equação do calor, também uma função $h_{2}(T)$

$$
h_{2}(T)=h\left(T-T_{0}\right)
$$

onde $h$ é dado pela relação 2.9 , onde aparece a viscosidade $\mu_{f}(T)$ do gás cirunjacente, para o qual não conhecemos uma expressão analítica e usamos dados tabelados.

Esta constatação nos leva a estabelecer alguns postulados acerca do modelo do capítulo 2.

Admitados que todas as relações não dadas analíticamente do capítulo 2 tem regularidade tal que os termos do SMRD do capítulo 2 satisfazem as condições 2, 3 e 4 de regularidade do seção 3.1.1. Quanto as relações dadas analiticamente, pelo menos para temperaturas $T \geq T_{1}$, para algum $T_{1}>0$, são todas $C^{\infty}$; por exemplo, $k$ da hematita em função de $T$ é uma expressão do tipo $\frac{1}{a T+b}$ que é $C^{\infty}$ em $T$ se $T \geq T_{1}$.

Sabemos que para temperaturas $T \leq T_{R}$ a mistura que forma a pelota é não-reativa e portanto todos os termos $\mathcal{R}$ se anulam. Daí a equação de balanço de calor fica desacoplada das equações de balanço de massa tomando a forma

$$
\frac{\partial T}{\partial t}=\operatorname{div} k_{s}^{\prime}(T) \operatorname{grad} T
$$

onde $k_{s}^{\prime}$ é $k_{s}$ multiplicada por alguma constante (seçao 2.3.1).

\footnotetext{
${ }^{2}$ A prova de que é $C^{m, 1}$ resulta de que $\frac{d h^{\prime}}{d T^{\prime}}$ é limitada em limitados de $\mathbb{R}$, qualquer $l$, e da aplicaçào do teorema do valor médio.
} 
Através de argumentos do príncípio do máximo (apêndiceA) aplicado à equação acima com condição de fronteira (2.8) temos que a temperatura $T$ satisfaz $T \geq T_{0}$, supondo perfil inicial de temperatura $T\left(t_{0}, x\right) \geq T_{0}$,para algum $T_{0}$ que aqui é a temperatura ambiente. Resulta que os termos de difusividade $k$ e condutividade $D^{j}$ do modelo físico satisfazem a condição de parabolicidade 1 da seção 3.1.1.

Segue destas considerações e da proposição 4.2.2 que o o SMRD acima admite uma única solução clássica local no tempo. 


\section{Capítulo 5}

\section{Conclusão}

Neste trabalho propusemos condições matemáticas simples para garantir existência e unicidade de solução clássica local no tempo a uma certa classe de sistemas parabólicos quaselineares com condição de fronteira não-linear a qual inclui, por exemplo, um modelo matemático de um problema de reaçãodifusão da engenharia química.

Usamos os resultados de Sobolevskiü na sua forma original sem usar a teoria de espacos de extrapolação de Amann, ainda assim obtemos um teorema de existência de solução clássica com condições essencialmente iguais às requeridas por ele [6].

Parece que o ponto de vista que adotamos, apesar de ser aplicável a uma classe mais restrita de problemas do que a teoria de Amann, talvez possibilite mais facilidade de aplicação de resultados abstratos de diferenciabilidade em relação ao dado inicial e talvez seja mais viável ao cálculo de soluções numéricas. 


\section{Apêndice A}

\section{Aplicação do princípio do máximo}

O teorema abaixo e sua prova são devidos a Henry.

Teorema A.0.3 Sejam $\Omega \subset \mathbb{R}^{n}$ aberto. limitado com fronteira de classe $C^{2}$, reais $T_{0}<T_{1},-\infty \leq m_{0}<m_{1} \leq+\infty$ e as funçôes. com $1 \leq i, j \leq n$.

$$
a_{i j}=a_{j i}, b_{i} \text { contínuas } \epsilon m \bar{\Omega} \times\left[T_{0} . T_{1}\right] \rightarrow \mathbb{R}
$$

com

$$
\sum_{i, j=1}^{n} a_{i j}(t . x) \xi_{i} \xi_{j}>0 \text { sempre que } \xi \neq 0 \text { em } \mathbb{R}^{n}
$$

e também as funçòes f.g com

$$
\begin{aligned}
& f \text { contínua em } \bar{\Omega} \times\left[T_{0}, T_{1}\right] \times \mathbb{R} \rightarrow \mathbb{R} \\
& g \text { contínua em } \partial \Omega \times\left[T_{0}, T_{1}\right] \times \mathbb{R} \rightarrow \mathbb{R}
\end{aligned}
$$

tais que

$s \geq m_{1}$ (se finito) $\Rightarrow f(t, x, s) \leq 0 \in g(t, x, s) \geq 0\left(t, x \in \bar{\Omega} \times\left[T_{0}, T_{1}\right] ; t, x \in \partial \Omega \times\left[T_{0} . T_{1}\right]\right)$ $s \leq m_{0}($ se finito $) \Rightarrow f(t, x, s) \geq 0$ e $g(t, x, s) \leq 0\left(t, x \in \bar{\Omega} \times\left[T_{0}, T_{1}\right] ; t, x \in \partial \Omega \times\left[T_{0} . T_{1}\right]\right)$

Seja $u(t, x)$ contínua em $\bar{\Omega} \times\left[T_{0} . T_{1}\right)$ com $u_{t}, u_{x}$ e $u_{x_{i} x}$, contínuas em $\Omega \times\left(T_{0}, T_{1}\right)$ e $u_{x_{i}}$ ainda contínua $\mathrm{em} \bar{\Omega} \times\left(T_{0}, T_{1}\right)$. 
Se

$$
\begin{aligned}
u_{t}= & \sum_{1 \leq i, j \leq n} a_{i j} u_{x_{i} x_{j}}+\sum_{i=1}^{n} b_{i} u_{x_{i}}+f(t, x, u) \text { em } \Omega \times\left(T_{0}, T_{1}\right) \\
& -\sum_{\substack{1 \leq i, j \leq n\\
}} a_{i j} N_{i} u_{x_{j}}=g(t, x, u) \text { em } \partial \Omega \times\left(T_{0}, T_{1}\right) \\
& {[N \text { normal exterior a } \partial \Omega \text { em cada ponto } x] }
\end{aligned}
$$

e

$$
m_{0} \leq\left. u\right|_{t=T_{0}} \leq m_{1} \text { em } \Omega
$$

então

$$
m_{0} \leq u \leq m_{1} \text { em } \bar{\Omega} \times\left[T_{0}, T_{1}\right) .
$$

Prova. Sem perda de generalidade, seja $\Omega$ conexo: caso contrário, tratamos cada componente separadamente.

Suponhamos $u>m_{1}$ em algum ponto - podemos mudar $T_{0}$, se necessário, e supor que isto vale arbitrariamente perto de $T_{0}$. Para $t^{0}>T_{0}$ pequeno e certo $x^{0} \in \bar{\Omega}, \max u_{\bar{\Omega} \times\left[T_{0}, t^{0}\right)}=u\left(P^{0}\right)>m_{1}, P^{0}=\left(t^{0}, x^{0}\right)$ e então por continuidade $u \geq m_{1}$ em viz $\left(P^{0}\right) \cap\left[T_{0}, t^{0}\right]$. Portanto $f \leq 0, g \geq 0$ em $(t, x, u(t, x))$ com $t, x$ nesta vizinhança e temos $u_{t} \leq A u$, onde $A u=\sum a_{i j} u_{x_{i} x_{j}}+\sum b_{i} u_{x_{i}}$ - Se $x^{0} \in \Omega$ temos $u \equiv u\left(P^{0}\right)>m_{1}$ numa viz $\left(P^{0}\right) \times\left[T_{0}, t^{0}\right] ; \operatorname{com} \Omega$ é conexo, temos. pelo princípio do máximo forte ([21], teorema 5, p.173), $u \equiv u\left(P^{0}\right)$ em todo $\bar{\Omega} \times\left[T_{0}, t^{0}\right]$ e chegamos a uma contradição com o valor inicial $\left.u\right|_{\left\{T_{0}\right\}} \times \Omega \leq m_{1}$.

Então suponhamos $u<u\left(P^{0}\right)$ em $\Omega \times\left[T_{0}, t^{0}\right)$ com $P^{0} \in \partial \Omega$ : temos, pelo princípio do máximo na fronteira $\left([21]\right.$, teorema 6 , p. 174), $\frac{\partial u}{\partial \nu}\left(P^{0}\right)>0$ $\left(\nu=\sum_{j} a_{i j} N_{j}\right)$, o que diz que $g\left(P^{0}, u\left(P^{0}\right)\right)<0$, que também é falso.

Assim não pode haver $u>m_{1}$ em qualquer ponto de $\bar{\Omega} \times\left[T_{0}, T_{1}\right)$. Da mesma maneira, $u<m_{0}$ é impossível.

Observação A.0.4 Os valores de $(f, g)(t, x, s)$ com $s \notin\left[m_{0}, m_{1}\right]$ são irrelevantes e $f, g$ podem se definidas de forma conveniente fora de $\left[m_{0}, m_{1}\right]$. Por exemplo, se $a_{i j}(t, x)=\hat{a}_{i j}(t, x, u(t, x))$, isto não tem importância se $\hat{a}_{i j}(t, x, s)>0$ para todo $m_{0} \leq s \leq m_{1} \in(t, x) \in\left[T_{0}, T_{1}\right] \times \bar{\Omega}$. 


\section{Referências Bibliográficas}

[1] O.A.Ladyzenskaya, V.A.Solonnikov e N.N.Ural'ceva, "Linear and Quasilinear Equation of Parabolic Type", Am. Math.Soc., Providence, Rhode Island (1968), Transl. of Math. Monographs 23.

[2] O.A.Ladyzenskaya e N.N.Ural'ceva, "Équations aux Dérivées Partielles de Type Elliptique", Monographies Universitaires de Mathématiques, Dunod, Paris, 1968.

[3] P.C. Fife, " Mathematical Aspects of Reacting and Diffusing Systems", Lectures Notes in Biomathematics, 28, Springer-Verlag, New York, 1979.

[4] F.Rothe, "Global Solutions of Reaction-Diffusion Systems", Lectures Notes in Mathematics, 1072, Springer-Verlag, New York/Tokyo, 1984.

[5] D. Henry, "Geometric Theory of Semilinear Parabolic Equations", Lectures Notes in Mathematics, 840, Springer-Verlag, New York/Berlin, 1981.

[6] H.Amann, " Dynamic Theory of Quasilinear Parabolic Equations. II. Reaction-Diffusion Systems", Differential And Integral Equations, 3 (1), (1990), 13-75.

[7] H.Amann, "Dynamic Theory of Quasilinear Parabolic Systens III. Global Existence", Mathematishe Zeitschrift, 202(2) (1989), 219-254.

[8] P.E.Soboleviskiı̌" Equation of Parabolic Type in a Banach Space" , Trans. Am. Math. Soc. 259,(1966), 547-557.

[9] D. Henry, "Some Infinite-Dimensional Morse-Smale Systems Defined by Parabolic Partial Differential Equations", Journal Differential Equations 59 (1985), 165-205. 
[10] A.Friedmann e B.MacLeod, "Blow-up of solutions of nonlinear parabolic equations. Arch. Rat. Mech. Anal., 96 (1986), 55-80.

[11] A.Friedman, "Partial Diferential Equations", Holt, Rinehart and Winston, New York (1969).

[12] A.Friedman, " Partial Differential Equations of Parabolic Type" , Prentice-Hall, Englewood Cliffs, New Jersey (1964).

[13] R.A.Adams, "Sobolev Spaces", Academic Press, New York (1975).

[14] H.Tanabe, "Equations of Evolution." Pitman, London (1979).

[15] M.B.Mourão, “ Análise do processo de redução de minério de ferro por carbono na forma de pelotas auto-redutoras", Tese de doutoramento, Escola Politécnica da Universidade de São Paulo, 1988.

[16] Julian Szekely, James W. Evans, Hong Yong Sohn, " Gas-Solid Reactions" , Academic Press, New York/São Francisco/Londres (1976).

[17] S.Sun e W-K. Lu, " Mathematical modelling of reactions in iron ore/coal composities", ISIJ International,bf 33 (10) (1993), 1062-1069.

[18] T.Akiyama, H.Ohta,R. Takahashi,Y.Waseda e J.Yagi, " Measurement and modeling of thermal conductivity for dense iron oxide and porous iron ore aglomerates in stepwise readuction" ,ISIJ International, 32 (7) (1992).

[19] R.B.Bird, W.E.Stewart e E.N.Lightfoot, "Transport Phenomena" , Wiley, New York (1960).

[20] G.H.Geiger e D.R.Poirier, "Transport Phenomena in Metallurgy", Addison-Wesley Publishing Company, Massachusetts (1973).

[21] Murray H.Protter e Hans F.Weinberger, "Maximun Principles in Differential Equations” ,Printice-Hall Inc., Englewood Cliffs, London (1967). 